\title{
ASYMPTOTIC GIANT BRANCH STARS AS A SOURCE OF SHORT-LIVED RADIOACTIVE NUCLEI IN THE SOLAR NEBULA
}

\author{
G. J. Wasserburg, ${ }^{1}$ M. Busso, ${ }^{2}$ R. GAllino, ${ }^{3}$ And C. M. RAiteri ${ }^{2}$ \\ Received 1993 June 11; accepted 1993 September 17
}

\begin{abstract}
We carried out a theoretical evaluation of the contribution of Asymptotic Giant Branch (AGB) stars to some short-lived $\left(10^{6} \leq \bar{\tau} \leq 2 \times 10^{7} \mathrm{yr}\right)$ isotopes in the ISM and in the early solar system using stellar model calculations for thermally pulsing evolutionary phases of low-mass stars. The yields of s-process nuclei in the convective He-shell for different neutron exposures $\tau_{0}$ were obtained, and AGB stars were shown to produce several radioactive nuclei (especially ${ }^{107} \mathrm{Pd},{ }^{205} \mathrm{~Pb},{ }^{60} \mathrm{Fe},{ }^{93} \mathrm{Zr},{ }^{99} \mathrm{Tc},{ }^{135} \mathrm{Cs}$, and ${ }^{182} \mathrm{Hf}$ ) in different amounts. Assuming either contamination of the solar nebula from a single AGB star or models for continuous injection and mixing from many stars into the ISM, we calculate the ratios of radioactive to stable nuclei at the epoch of the Sun's formation. The dilution factor between the AGB ejecta and the early solar system matter is obtained by matching the observed ${ }^{107} \mathrm{Pd} /{ }^{108} \mathrm{Pd}$ and depends on the value of $\tau_{0}$. It is found that small masses $M_{\mathrm{He}}$ of He-shell material $\left(10^{-4}-10^{-7} M_{\odot}\right)$ enriched in s-process nuclei are sufficient to contaminate $1 M_{\odot}$ of the ISM to produce the ${ }^{107} \mathrm{Pd}$ found in the early solar system. Predictions are made for all of the other radioactive isotopes. The optimal model to explain several observed radioactive species at different states of the proto-solar nebula involves a single AGB star with a low neutron exposure $\left(\tau_{0}=0.03 \mathrm{mbarn}^{-1}\right)$ which contaminated the cloud with a dilution factor of $M_{\mathrm{He}} / M_{\odot} \sim 1.5 \times 10^{-4}$. This will also contribute newly synthesized stable s-process nuclei in the amount of $\sim 10^{-4}$ of their abundances already present in the protosolar cloud. Variations in the degree of homogenization $(\sim 30 \%)$ of the injected material may account for some of the small general isotopic anomalies found in meteorites. It is also found that ${ }^{60} \mathrm{Fe}$ is produced in small but significant quantities that may be sufficient to explain the observations if the time elapsed $(\Delta)$ from the contamination of the ISM to the formation of protoplanetary bodies is not higher than $\Delta=5 \times 10^{6} \mathrm{yr}$. If $\Delta$ is longer, up to $10 \times 10^{6} \mathrm{yr}$, this would require the single AGB star to experience enhanced neutron densities $\left(n_{n} \sim 3 \times 10^{9} n \mathrm{~cm}^{-3}\right)$ in the s-processing zone in order to compensate for the branching at ${ }^{59} \mathrm{Fe}$. The alternative model of long-term continuous ejection of matter from many AGB stars does not appear to match the observations. We also estimate the ${ }^{26} \mathrm{Al}$ production from the $\mathrm{H}$-shell and find that the ${ }^{26} \mathrm{Al}$ abundance in the early solar system may be readily explained in a self-consistent manner. Moreover, ${ }^{26} \mathrm{Al}$ from AGB stars may contribute substantially to the galactic ${ }^{26} \mathrm{Al} \gamma$-source, while no significant $\gamma$-flux from ${ }^{60} \mathrm{Co}$ (deriving from ${ }^{60} \mathrm{Fe}$ decay) is to be expected.
\end{abstract}

Subject headings: ISM: abundances - nuclear reactions, nucleosynthesis, abundances solar system: formation - stars: AGB and post-AGB

\section{INTRODUCTION}

The purpose of this study is to estimate the possible contribution of short-lived nuclei to the solar nebula from red giants that undergo recurrent He-shell thermal pulses on the asymptotic giant branch phase (hereafter TP-AGB). In these evolutionary stages important neutron fluxes can be released by $\alpha$ captures either on ${ }^{22} \mathrm{Ne}$ or on ${ }^{13} \mathrm{C}$ (Truran \& Iben 1977; Iben \& Renzini 1982). As first noticed by Ulrich (1973), the recurrence of thermal pulses can naturally account for an exponential distribution of neutron exposures: $\rho(\tau) \propto \exp \left[-\tau / \tau_{0}\right]$, where $\tau=\int n_{n}(t) v(t) d t$ is the time-integrated neutron flux, $n_{n}$ is the neutron density, and $v$ is the thermal velocity. This is required to reproduce the main $s$-process component in the solar system that accounts for the $s$-nuclei in the mass range $90 \leq A \leq 209$ (see Käppeler, Beer, \& Wisshak 1989 for a

\footnotetext{
${ }^{1}$ Lunatic Asylum, Division of Geological and Planetary Sciences, 170-25, California Institute of Technology, Pasadena, CA 91125.

${ }_{2}$ Osservatorio Astronomico di Torino, Strada Osservatorio 20, 10025 Pino Torinese, Italy.

${ }^{3}$ Istituto di Fisica Generale dell'Università, Via P. Giuria 1, 10125 Torino, Italy.
}

recent discussion). With an up to date nuclear network, the best-fit mean neutron exposure is $\tau_{0}=(0.28 \pm 0.01)$ mbarn $^{-1}$ (Gallino, Raiteri, \& Busso 1993).

There is abundant observational evidence that most TP-AGB stars are of low mass $\left(1\right.$ to $\left.3 M_{\odot}\right)$ and are enriched in carbon and in $s$-process elements and are undergoing efficient mass loss by stellar winds (Smith \& Lambert, 1985, 1986, 1990; Utsumi 1985; Lambert 1991). Nucleosynthesis calculations have shown that the $s$-processing that takes place in these giants requires ${ }^{13} \mathrm{C}$ as the major neutron source (Busso et al. 1988; Hollowell \& Iben 1988, 1989; Gallino et al. 1988; Käppeler et al. 1990). Indeed, the temperature at the bottom of the convective He-shell remains low $\left(T_{8} \sim 1.5\right)$ for the majority of the pulse duration, and reaches $T_{8}=3$ only at the end, when the thermal instability grows suddenly to its maximum extension, so that the alternative ${ }^{22} \mathrm{Ne}$ neutron source is not activated efficiently. This fact has received clear observational support (Tomkin \& Lambert 1979; Clegg, Lambert, \& Bell 1979). However, the formation of the neutron source ${ }^{13} \mathrm{C}$ depends on the difficulty in treating the partial mixing at the $\mathrm{H}-\mathrm{He}$ interface. No self-consistent model for this process has yet been found in stellar models with metallicity near the solar one. Consequently, in order to follow the $s$-processing in TP-AGB 
stars, the amount of ${ }^{13} \mathrm{C}$ to be ingested by each thermal instability has to be assumed parametrically (see Busso et al. 1992 for a discussion).

Together with ${ }^{12} \mathrm{C}$ and $s$-process isotopes from He-burning layers, TP-AGB stars also give important contributions of $\mathrm{H}$ burning products. These include ${ }^{14} \mathrm{~N}$, mixed to the surface after the main sequence in the first dredge-up (Renzini \& Voli 1981), and the radioactive isotope ${ }^{26} \mathrm{Al}$, produced in the AGB by proton captures on ${ }^{25} \mathrm{Mg}$ in high-temperature $\mathrm{H}$-shell conditions, as first noticed by Forestini, Paulus, \& Arnould (1991). The ${ }^{26} \mathrm{Al} /{ }^{27} \mathrm{Al}$ ratio they obtain in the stellar envelope is in the range of those measured in interstellar $\mathrm{SiC}$ grains found in some meteorites (Zinner et al. 1991; Huss, Hutcheon, \& Wasserburg 1993; Hoppe et al. 1994). We will not discuss here the important results on individual interstellar grains, but will focus on global values for the solar nebula.

In this study we will reanalyze the whole problem of the origin of short-lived nuclides from AGB sources. Previous quantitive estimates in AGB environments were limited either to $s$-process species, using a simplified steady-flow approximation for the neutron exposure (Cameron et al. 1993), or to the evaluation of $\mathbf{H}$-shell products (Forestini et al. 1991). These calculations do not give a set of yields of all the different isotopes but typically give isotope ratios for a single element for steady state flow patterns. There is now the need for a more comprehensive analysis that follows AGB evolution, and takes into account the net production of all nuclei both from $\mathrm{H}$ burning and He-burning layers, including the dilution effects of mixing of nuclear-processed matter first to the surface of the star, through repeated episodes of convective penetration from the envelope, and secondly, into the interstellar medium (hereafter ISM) through stellar winds. Our efforts are directed toward calculating the isotopic abundances of all pertinent radioactive nuclei produced in AGB stars for different dilution mechanisms. This provides a framework for predicting abundances of several nuclei that have not yet been observed as well as explaining some species that have been discovered.

An extensive discussion of all the interesting radioactive species in the early solar system and of their possible astrophysical origin was recently presented by Cameron (1993). Here we shall restrict our attention to those nuclei that can receive non-negligible contributions either from $s$-processing (e.g., ${ }^{107} \mathrm{Pd},{ }^{205} \mathrm{~Pb},{ }^{60} \mathrm{Fe}$ ), or from H-burning $\left({ }^{26} \mathrm{Al}\right)$ in AGB conditions. The relevant isotopes are summarized in Table 1. We will not discuss those nuclei $\left({ }^{244} \mathrm{Pu},{ }^{53} \mathrm{Mn}\right)$ which, while well-identified, cannot come from AGB sources (Podosek \& Swindle 1988; Birck \& Allègre 1985).

TABLE 1

MEAN LiVes OF THE Isotopes STUdied

\begin{tabular}{cccc}
\hline \hline Nuclide & $\begin{array}{c}\bar{\tau}_{R} \text { (yr) } \\
\text { (Laboratory) }\end{array}$ & $\begin{array}{c}\bar{\tau}_{R}(\mathrm{yr}) \\
\left(T_{8}=1.5\right)\end{array}$ & $\begin{array}{c}\bar{\tau}_{R}(\mathrm{yr}) \\
\left(T_{8}=3.0\right)\end{array}$ \\
\hline${ }^{107} \mathrm{Pd} \ldots \ldots \ldots \ldots$. & $9.4 \times 10^{6}$ & $7.0 \times 10^{4}$ & $1.1 \times 10^{3}$ \\
${ }^{205} \mathrm{~Pb} \ldots \ldots \ldots \ldots$. & $2.2 \times 10^{7}$ & 5.17 & 7.05 \\
${ }^{60} \mathrm{Fe} \ldots \ldots \ldots \ldots \ldots$ & $2.2 \times 10^{6}$ & $2.2 \times 10^{6}$ & $2.2 \times 10^{6}$ \\
${ }^{93} \mathrm{Zr} \ldots \ldots \ldots \ldots \ldots$ & $2.2 \times 10^{6}$ & $2.2 \times 10^{6}$ & $2.2 \times 10^{6}$ \\
${ }^{99} \mathrm{Tc} \ldots \ldots \ldots \ldots \ldots$ & $3.1 \times 10^{5}$ & 320 & 6.5 \\
${ }^{135} \mathrm{Cs} \ldots \ldots \ldots \ldots \ldots$ & $4.3 \times 10^{6}$ & $6.0 \times 10^{5}$ & 400 \\
${ }^{182} \mathrm{Hf} \ldots \ldots \ldots \ldots$. & $1.3 \times 10^{7}$ & $3.0 \times 10^{3}$ & 26 \\
${ }^{129} \mathrm{I} \ldots \ldots \ldots \ldots \ldots$ & $2.3 \times 10^{7}$ & 6 & 0.8 \\
${ }^{26} \mathrm{Al} \ldots \ldots \ldots \ldots \ldots$ & $1.1 \times 10^{6}$ & $1.1 \times 10^{6}$ & $1.1 \times 10^{6}$ \\
\hline
\end{tabular}

In $\S 2$ we briefly review the main characteristics of the models; in $\S 3$ a technique is described for calculating the expected isotopic ratios of radioactive to stable species in the early solar nebula for different models. This technique is then applied in $\S 4$ to all the unstable nuclei studied here, deriving the required dilution factors of the stellar material for the cases of a single late addition of AGB products and continuous injection over a time $t$. In this section, these radioactive isotopic abundances and dilution factors are compared with those expected from typical ISM conditions at the moment of the solar nebula formation but, for simplicity, without considering solar system timescales. In $\S 5$ we then discuss the problem of timescales between late addition, early solar nebular condensate formation and the formation of early solar system planetary bodies. Finally, the main conclusions are summarized in $\S 6$. A preliminary report of this study was presented earlier (Wasserburg et al. 1993).

\section{STELLAR MODEL CALCULATIONS}

The approach taken here is to evaluate the production of nuclei for a star of initial mass in the range 1.5 to $3 M_{\odot}$ and solar composition during the TP-AGB phase of evolution. Detailed models for 1.5 and $3 M_{\odot}$ stars were performed recently (Busso et al. 1993). The models were started from the main sequence and prosecuted up to the end of the TP-AGB. Details on the computations will be published elsewhere (Chieffi, Limongi, \& Straniero 1994); here we shall only discuss the general features of the stellar structure during the thermally pulsing phase, where the nuclei of interest in this paper can be produced.

The inner portion of the star is in the form of a degenerate $\mathrm{C}-\mathrm{O}$ core of growing mass (from about $0.50 \mathrm{M}_{\odot}$ at the first pulse, up to about 0.7 at the end, when the star leaves the AGB phase). Outside this core, a He-burning shell and a H-burning shell are alternatively active, controlling the stellar luminosity. For the majority of time, it is the H-burning shell which operates efficiently, advancing monotonically in mass for long intervals (the so-called interpulse periods, of duration declining with increasing pulse number, from $10^{5}$ yr down to $3-4 \times 10^{4}$ yr). The whole core over which hydrogen is exhausted will be referred to as $M_{\mathrm{C}}$. Meanwhile, the position in mass of the He-shell doesn't vary appreciably. Due to increasing compression from the overlying $\mathrm{H}$-burning, the intershell region is periodically swept by episodes of convective He-burning (the thermal pulses, or thermal instabilities). The relevant features of the stellar structure during these evolutionary stages are sketched in Figure 1, where the pulses appear as vertical lines due to their short duration in time ( $300 \mathrm{yr})$ as compared to the interpulse periods. When the convective instability reaches its maximum extension, it covers almost the whole intershell layer, except for a thin interval (labeled as " $\mathrm{A}$ " in the figure) whose mass is a decreasing function of $M_{\mathrm{C}}$, from $1-2 \times 10^{-3}$ $M_{\odot}$ at the first cycles down to about $10^{-4} M_{\odot}$ in the final ones. Immediately after the pulse, the outer layers of the star are forced to expand and cool, so that the H-burning shell becomes temporarily extinguished and the envelope convection may extend downward, dredging up to the surface newly synthesized nuclei produced by $\mathbf{H}$ and $\mathrm{He}$ burning. In the 3 $M_{\odot}$ model, this dredge-up was found to occur after the 14 th pulse, at a core mass $M_{\mathrm{C}}=0.65 M_{\odot}$, and to repeat regularly from then on, involving on average about $10^{-3} M_{\odot}$ per pulse. In the material which is thus mixed to the envelope (labeled as "B" in Fig. 1), we can distinguish two parts of different com- 


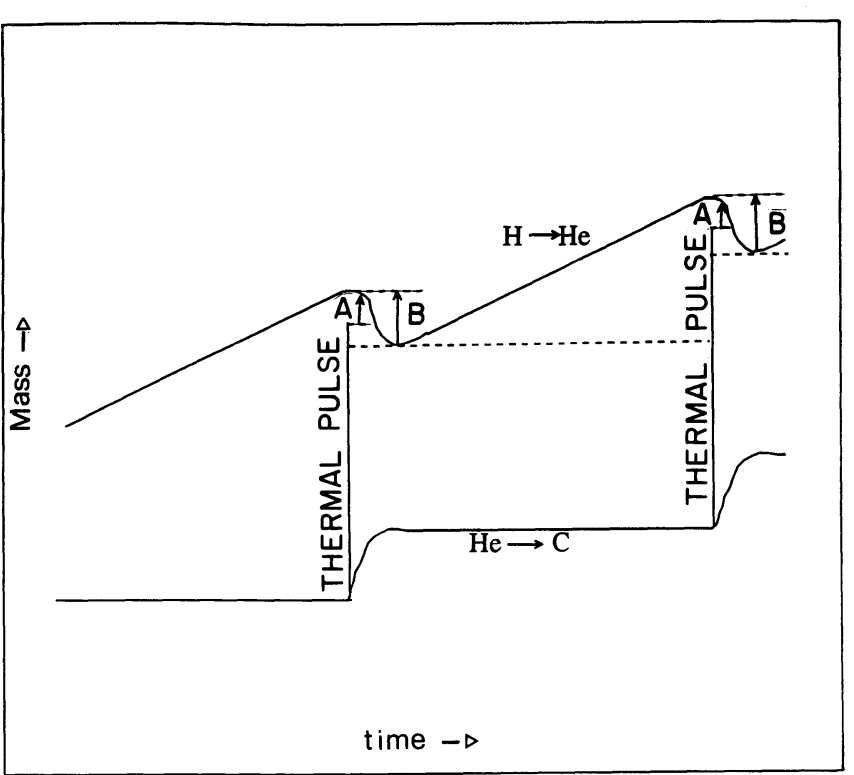

FIG. 1.-Schematic view of the stellar region of interest for our calculations of nucleosynthesis in the TP-AGB phases. Lower and upper curves represent position in mass coordinates of the burning $\mathrm{He}$-shell (that overlies the $\mathrm{C}-\mathrm{O}$ core) and H-burning shell (that lies at the base of the convective envelope), respectively, as a function of time (see text for details). Region "A" is in the He zone but outside of region of convective instability. Region " $\mathrm{B}$ " is mixed into the envelope.

position. The layer " $A$ " contains only H-burning ashes, while the inner part includes He-burning products like $\mathrm{C}$ and $s$-process nuclei. We shall refer to the whole mass mixed to the envelope (in several dredge-up episodes) from region " $A$ " $(H-$ exhausted) as $M_{\mathrm{H}}$; that mixed from the underlying region that has seen a partial He-burning, will be identified as $M_{\mathrm{He}}$.

It has been suggested (Iben \& Renzini 1982; Hollowell \& Iben 1988,1989$)$ that for halo stars a small semiconvective region driven by partial carbon recombination in the expanding material, can develop below the border of envelope convection when this reaches it maximum downward extension, thus mixing a small amount of protons from the envelope into the $\mathrm{C}$-rich zone. As soon as the star contracts again and heats, these protons are captured by ${ }^{12} \mathrm{C}$ producing fresh ${ }^{13} \mathrm{C}$ and some ${ }^{14} \mathrm{~N}$ in a tiny pocket. This mechanism does not work in stars of solar metallicity (Iben 1983); however, a thin interface layer unstable against semiconvective mixing is usually found in the models (see also Boothroyd \& Sackmann 1988). In this layer different authors either inhibit mixing or allow it to occur completely (convectively), because of the difficulties in treating the boundary layer physics problems. A proper treatment of the ${ }^{13} \mathrm{C}$ pocket formation should reanalyze this problem with time-dependent mixing algorithms. In the absence of a suitable model, we are still forced to vary parametrically the amount of ${ }^{13} \mathrm{C}$ which is ingested by each pulse. Values in the range from 1 to $10 \times 10^{-6} M_{\odot}$ are considered here. This pocket is then ingested by the following convective pulse, when this expands to its maximum amplitude; the region where the ingestion occurs is identified in Figure 1 by a dashed horizontal line.

The mass of ${ }^{13} \mathrm{C}$ burnt fixes the neutron exposure per pulse $\Delta \tau$; this and the overlapping factor $r$ (i.e., the fraction of the pulse mass which has already been processed in a previous convective instability) fix the asymptotic neutron exposure $\tau_{0}$, according to the law $\tau_{0}=\Delta \tau /[-\ln (r)]$ (Ulrich 1973). The rate of ingestion of ${ }^{13} \mathrm{C}$ into the convective region and the rate of the ${ }^{13} \mathrm{C}(\alpha, n){ }^{16} \mathrm{O}$ reaction fix the average neutron density, $n_{n}$. Hence, in any model of $s$-processing in TP-AGB stars, it is essential to reproduce carefully the pulse physics and structure (temperature, density, ingestion rate) during ${ }^{13} \mathrm{C}$ burning and to use realistic values of $r$. In order to do this we have taken the relevant parameters from the stellar models, and then we have separately computed the nucleosynthesis in the convective regions, by modeling these last as shown in Figure 2, where a real pulse shape is superposed on the schematic structure we use for $s$-processing. The entire pulse duration is divided into four main phases: before ${ }^{13} \mathrm{C}$ ingestion (region number 1 in the figure), He-burning occurs without neutron captures, so that a proper reproduction of the convective growth is not essential. In zone number 2 the ingestion and burning of ${ }^{13} \mathrm{C}$ is, instead, modelled carefully: the actual position in mass of the ${ }^{13} \mathrm{C}$ pocket is deduced from the overlapping factor and the rate of convective expansion is matched to the real shape. In the third zone (no further ingestion), the consumption of the remaining ${ }^{13} \mathrm{C}$ occurs and, at the end, convection is assumed to expand instantaneously to its maximum size (again, since no neutrons are present, a schematic description is sufficient for this part). Finally, in the last phase, the bottom temperature sharply increases up to $2.8-3 \times 10^{8} \mathrm{~K}$ for a duration of about $2 \mathrm{yr}$ and then decreases exponentially. Here the neutron source ${ }^{22} \mathrm{Ne}(\alpha, n){ }^{25} \mathrm{Mg}$ is marginally activated. As a typical pulse shape and extension, we adopt those obtained at a core mass $M_{\mathrm{C}} \approx 0.60 M_{\odot}$. Though ${ }^{22} \mathrm{Ne}$ contributes only a minor part of the total neutron exposure, the last phase of the pulses involving this source is important for some nuclear effects. In particular, enhanced $\beta$ decays modify the abundances of those unstable species having a mean life which is a sensitive function of temperature. Examples of some of the lifetimes of the nuclei studied here are reported in Table 1 for some conditions prevailing in the models during ${ }^{13} \mathrm{C}$ and ${ }^{22} \mathrm{Ne}$ burning (cf. Takahashi \& Yokoi 1987). In each pulse the nucleosynthesis is followed through an extended network from He to Po. The neutron capture cross sections were taken from Beer, Voss, \& Winters (1992), while the weak interactions at astrophysical temperature and density conditions are from Fuller, Fowler, \& Newman (1982), and Takahashi \& Yokoi (1987). The initial isotopic and chemical abundances are from Anders \& Grevesse (1989) but the results are insensitive to the various tabulations of "cosmic" abundances (cf. Cameron 1984). Details of AGB calculations are described more extensively in Gallino et al. (1993) and Busso et al. (1992, 1994).

With the above assumptions, we computed the asymptotic values of the neutron exposures in the range 0.03 to 0.28 mbarn $^{-1}$. The asymptotic behavior is obtained within about 20 cycles. The enhancement factors of the abundances in the He-shell at the 20th cycle with respect to the initial solar composition are shown in Figures 3 to 6 , for all the relevant stable nuclei heavier than $A=56$, for the cases with $\tau_{0}=0.03,0.07$, $0.13,0.28$ mbarn $^{-1}$. Tables of the detailed nuclear abundances in the He-shell can be obtained from the authors on request. The last two cases $\left(\tau_{0}=0.13\right.$ and 0.28 mbarn $\left.^{-1}\right)$ were shown to be relevant for explaining some isotopic anomalies in meteoritic SiC grains and the main s-process component in the solar system, respectively (Gallino et al. 1993). The first two cases are new models, computed for this work.

We are also interested in the production of ${ }^{26} \mathrm{Al}$, which is efficiently produced by high-temperature $\left(T>3.5 \times 10^{7} \mathrm{~K}\right)$ H-burning, through proton captures on ${ }^{25} \mathrm{Mg}$. Here, an average concentration of ${ }^{26} \mathrm{Al}$ corresponding to $80 \%$ of the original ${ }^{25} \mathrm{Mg}$ is obtained. From the nucleosynthesis point of 

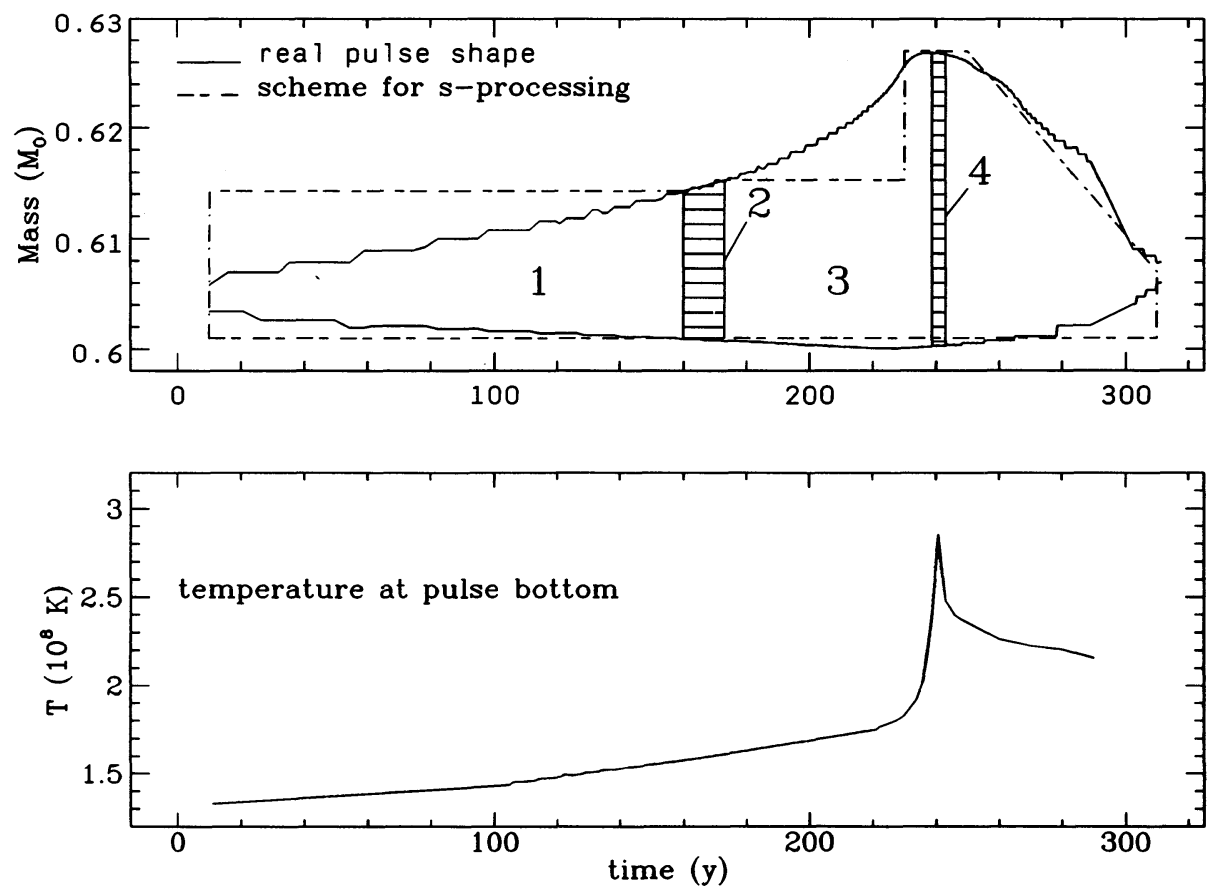

Fig. 2.-Top panel shows the actual calculated profile of a convective instability in the stellar evolution model (continuous line). The vertical extent in mass corresponds to the region of convective mixing. Region A of Fig. 1 lies above this region of convective mixing. Superposed on this is the schematic pulse shape of rectangles and truncated rectangles that we adopted in nucleosynthesis computations (dashed line). Region 1 is prior to ${ }^{13} \mathrm{C}$ ingestion and does not require a detailed description. In region 2 (horizontal lines) convective expansion engulfs the model ${ }^{13} \mathrm{C}$-pocket so that ${ }^{13} \mathrm{C}$ starts to produce neutrons. Here the maximum value of the neutron density is reached. In region 3 the residual ${ }^{13} \mathrm{C}$ abundance is simply burnt, producing a decreasing neutron density. Region 4 (horizontal lines) is where the marginal activation of the ${ }^{22} \mathrm{Ne}(\alpha, n)$ source occurs, due to the final sharp temperature increase. The temperature profile showing this increase is reproduced in the bottom panel. The zone following region 4 has no neutron sources.

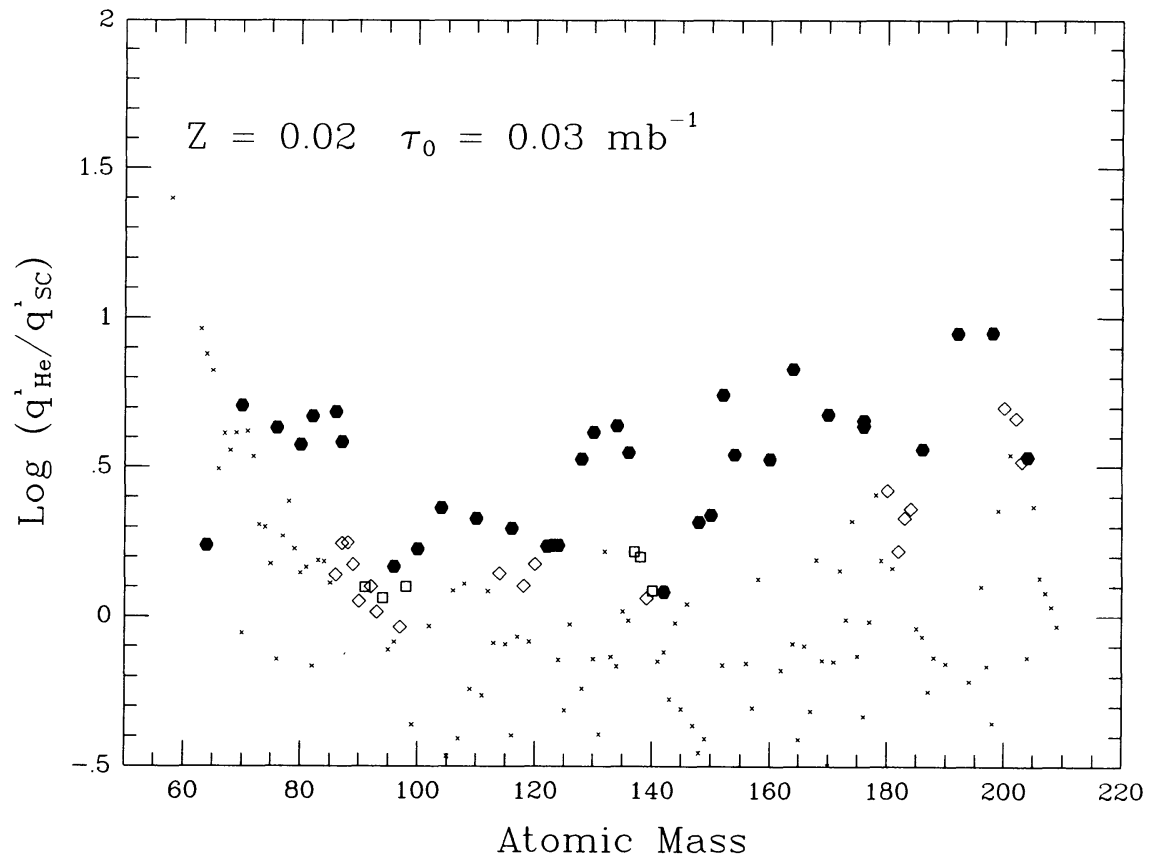

FIG. 3.-Distribution of enhancement factors in the He-shell $\left(q_{\mathrm{He}}^{i} / q_{\mathrm{SC}}^{i}\right)$ with respect to the initial composition for the case with $\tau_{0}=0.03 \mathrm{mbarn}^{-1}$. Full hexagons refer to $s$-only nuclei, open squares to nuclei with an $s$-process contribution in excess of $80 \%$, open rhombs to those with $s$-process contribution between $60 \%$ and $80 \%$. Crosses represent all the other isotopes. 


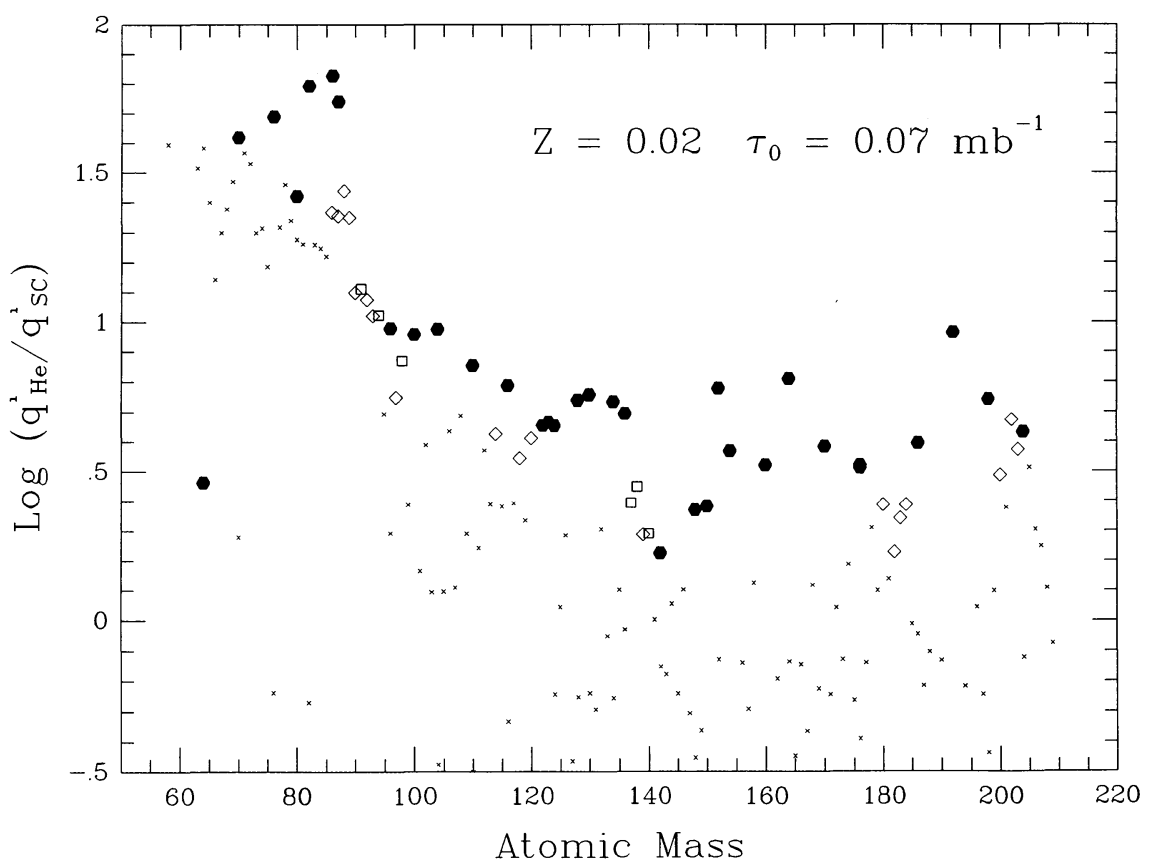

FIG. 4.-Same as Fig. 3, but for the case with $\tau_{0}=0.07 \mathrm{mbarn}^{-1}$

view, we confirm the results of Forestini et al. (1991), who first analyzed in detail the production of ${ }^{26} \mathrm{Al}$, using suggestions by Vogelaar (1989) and Iliadis et al. (1990) for reaction rates, and using models from Forestini et al. (1992). However, as the $\mathrm{H}$-shell moves outward in mass, this region where ${ }^{26} \mathrm{Al}$ was produced becomes part of the underlying He-zone. When this zone is swept by the subsequent thermal pulse, ${ }^{26} \mathrm{Al}$ efficiently suffers neutron captures [mainly $(n, p)$ reactions] due to the neutron fluxes released there. From this point of view, our calculations differ from those by Forestini et al. (1991), since the above authors did not consider the formation of the ${ }^{13} \mathrm{C}$ pocket and, consequently, did not follow in detail the $s$ processing in the convective He-burning zone. We find that the ${ }^{26} \mathrm{Al}$ contained in the pulse before its expansion to the maximum amplitude, is completely destroyed by the neutrons released by the ${ }^{13} \mathrm{C}$ neutron source (see Fig. 2). The amount of ${ }^{26} \mathrm{Al}$ engulfed subsequently by the growth of the convective instability in the last phase is first diluted over the entire con-

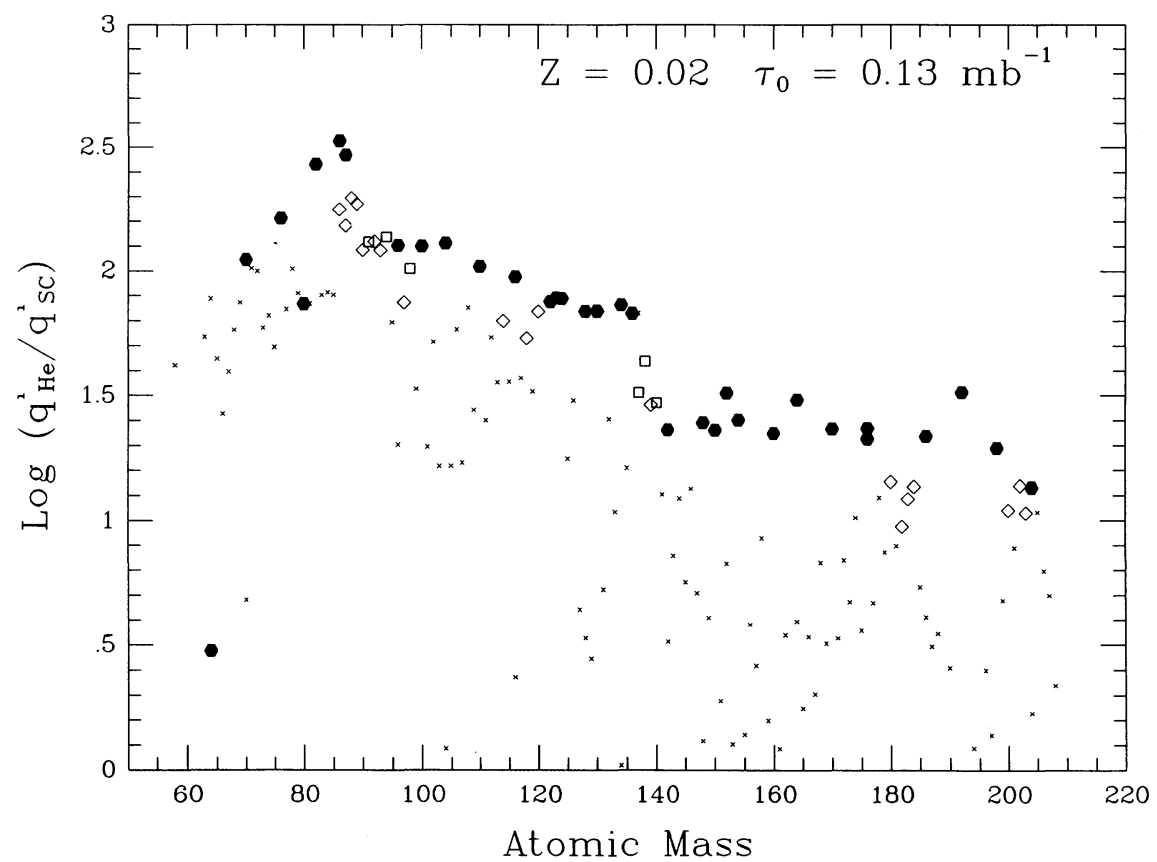

FIG. 5. - Same as Fig. 3, but for the case with $\tau_{0}=0.13 \mathrm{mbarn}^{-1}$. (Note change in scale.) 


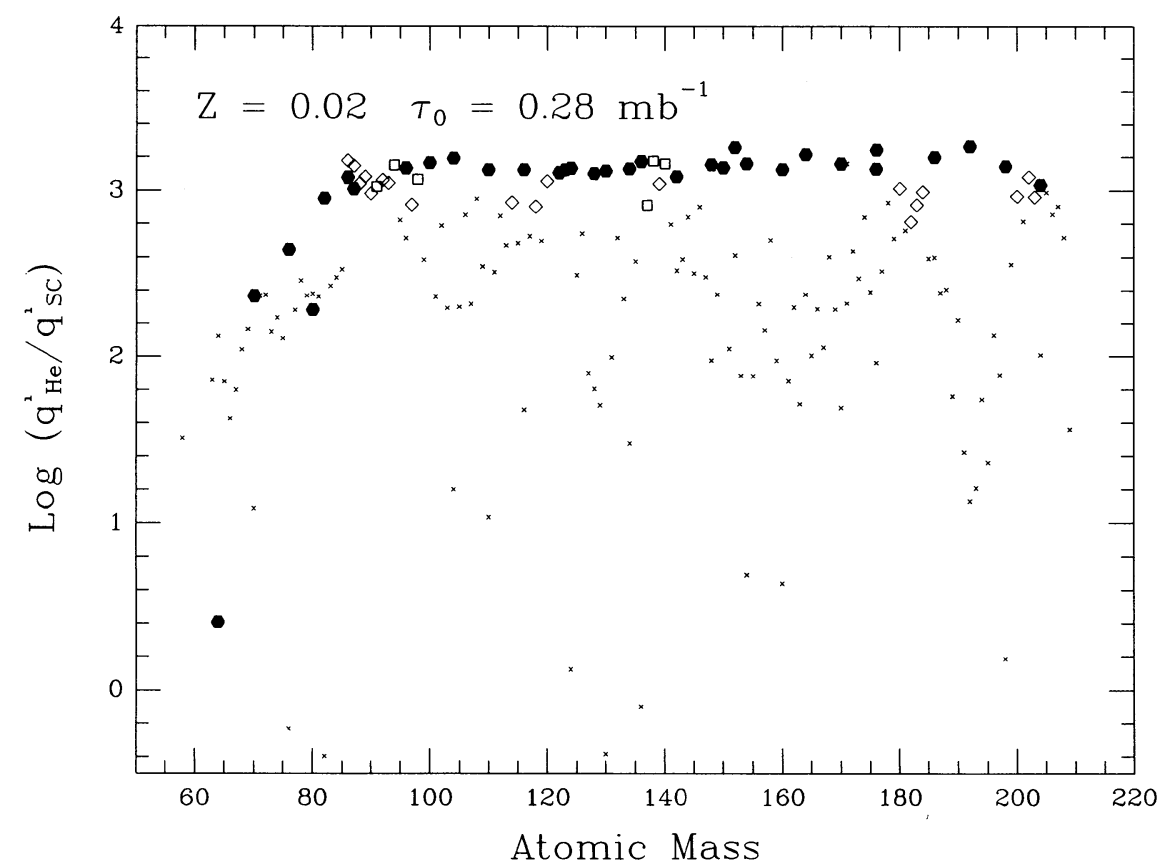

Fig. 6. -Same as Fig. 3, but for the case with $\tau_{0}=0.28 \mathrm{mbarn}^{-1}$

vective region and then undergoes $n$-captures due to the marginal activation of the ${ }^{22} \mathrm{Ne}$ neutron source. As a consequence, the ${ }^{26} \mathrm{Al}$ in the convective zone is destroyed by typically $\sim 80 \%$ (see also Gallino et al. 1994). Concerning the rates for the $(n, p)$ and $(n, \alpha)$ reactions (which are the main channels of destruction for ${ }^{26} \mathrm{Al}$ in He-burning conditions) we used the estimates by Caughlan \& Fowler (1988), assuming that the $0^{+}$isometric state is not thermalized with respect to the $5^{+}$ground state (Ward \& Fowler 1980). For the $(n, p)$ reaction, the Caughlan \& Fowler (1988) cross section is about $40 \%$ larger than the one measured by Trautvetter et al. (1989), which, however, was limited to the $p_{0}$ and $p_{1}$ transitions to the ground state and to the first excited state of ${ }^{26} \mathrm{Mg}$. In contrast to the ashes of the $\mathrm{H}$-shell that are mixed into the convective He-burning zone, that part of the H-shell which is left above the top of the convective He-shell is instead mixed directly to the surface in each dredge-up episode and has its ${ }^{26} \mathrm{Al}$ preserved.

As mentioned above, this region containing ${ }^{26} \mathrm{Al}$ (which was labeled " $A$ " in Fig. 1) is found to decrease in mass with increasing pulse number from about $10^{-3} M_{\odot}$ in the first pulses down to $10^{-4} M_{\odot}$. Its average value over the entire AGB duration is from 3 to $6 \times 10^{-4} M_{\odot}$, depending on the total number of pulses experienced (i.e., on the mass-loss rate adopted). Concerning the extension of this zone, our result is rather typical, as can be seen from current TP-AGB calculations (see e.g., Iben 1982; Hollowell \& Iben 1989; Lattanzio 1993). Henceforth we shall adopt for the mass of this zone the average value $5 \times 10^{-4} M_{\odot}$, with a probable uncertainty of a factor of 2 . In this respect, our treatment further differs from that of Forestini et al. (1991); indeed, these authors did not consider, in the dredge-up material, the important contribution to ${ }^{26} \mathrm{Al}$ from the pure He-region remaining above the thermal pulse.

In order to obtain the dilution factors achieved by newly synthesized species in the envelope, we mix the material from $\mathrm{He}$ - and $\mathrm{H}$-shells to the surface by assuming that convection penetrates by about $10^{-3} M_{\odot}$ into the carbon-enriched region of the core, as suggested by recent models (Lattanzio 1986; Chieffi et al. 1994; Busso et al. 1993). The procedure is repeated, pulse after pulse, until a carbon star with $\mathrm{C} / \mathrm{O} \sim 1.0$ in the envelope is obtained; this situation is then assumed to represent average envelope conditions during the whole AGB phase. With $\mathrm{C} / \mathrm{O} \sim 1$ this corresponds to a mixture of He shell matter $(25 \% \mathrm{C}$ by weight) with what was originally unprocessed envelope $(0.3 \% \mathrm{C}$ by weight $)$ of $M_{\mathrm{He}} / M_{\mathrm{E}} \sim$ $2 \times 10^{-2}$.

\section{ABUNDANCES OF RADIOACTIVE NUCLEI FROM AGB SOURCES}

We examine here those short-lived nuclei having lifetimes greater than $10^{6} \mathrm{yr}$ which may receive contributions from the $s$-process. Together with them we include ${ }^{26} \mathrm{Al}$, which can have a source in AGB stars (cf. Forestini, Paulus, \& Arnould 1991). Our approach is to ascertain the amounts of AGB ejecta necessary to provide each of these radioactive nuclei in the early solar nebula, looking for those that can be fully or partly accounted for in this way. Though the adopted neutron exposures range from a low value to the one that characterizes the main s-process component, we note that we are not attempting to reproduce the solar system abundances of stable isotopes. On the contrary, the amounts of material that are required here are small, since we only need enough freshly synthesized matter to salt the ISM with a late addition of short-lived species.

Consider that the mass of the solar nebula consists of material from two sources: matter from the pre-existing average ISM (which we shall refer to with the subscript $\mathrm{O}$ ) and freshly added matter from late stage additions from AGB stars. The AGB matter further contains contributions from the original composition of the convective envelope (subscript $E$ ) and from nucleosynthesis either by the He-burning shell (subscript $\mathrm{He}$ ) or, for ${ }^{26} \mathrm{Al}$, by the $\mathrm{H}$-burning shell (subscript $\mathrm{H}$ ). The total masses contributed by each reservoir are $M_{\mathrm{O}}, M_{\mathrm{E}}, M_{\mathrm{He}}$, and 
$M_{\mathrm{H}}$. Hence the mass of the proto-solar cloud (SC) is $M_{\mathrm{SC}}=$ $M_{\mathrm{O}}+M_{\mathrm{E}}+M_{\mathrm{He}}+M_{\mathrm{H}}$. Let the number of nuclei $i$ in each reservoir be given by $N_{P}^{i}$ where $P$ designates the reservoir $(\mathrm{O}$, $\mathrm{E}, \mathrm{He}, \mathrm{H})$. Let $q_{P}^{i}=N_{P}^{i} / M_{P}$ be the number of nuclei $i$ per gram in reservoir $P$. For most nuclei we may neglect $M_{\mathrm{H}}$ and include it in the term for $M_{\mathrm{E}}$. The ratio $q_{\mathrm{He}}^{I} / q_{\mathrm{SC}}^{I}$ is the enhancement factor of isotope $I$ in the $\mathrm{He}$ shell relative to the proto-solar cloud. Let us assume that the time scale for removal from the AGB and mixing with the SC is short compared with $10^{6} \mathrm{yr}$. For a radioactive species $R$ and for a stable index species $I$ of the same chemical element, both produced only in the He zone, we obtain: $N_{\mathrm{SC}}^{R}=N_{\mathrm{He}}^{R}$ and $N_{\mathrm{SC}}^{I}=N_{\mathrm{O}}^{I}+N_{\mathrm{He}}^{I}+N_{\mathrm{E}}^{I}$. If a time $\Delta$ of free decay is allowed, then all of the radioactive nuclei with mean life $\bar{\tau}_{R}$ will have their abundances in the cloud decreased by the factor $e^{-\Delta / \bar{\tau}_{R}}$. A discussion involving free decay will be presented later. For the initial solar nebula value $N_{\mathrm{SC}}^{R} / N_{\mathrm{SC}}^{I} \equiv$ $\alpha_{R, I}$, we obtain

$$
\alpha_{R, I}=\frac{N_{\mathrm{SC}}^{R}}{N_{\mathrm{O}}^{I}+N_{\mathrm{He}}^{I}+N_{\mathrm{E}}^{I}}=\frac{\left(N_{\mathrm{He}}^{R} / N_{\mathrm{He}}^{I}\right) q_{\mathrm{He}}^{I} M_{\mathrm{He}}}{q_{\mathrm{O}}^{I} M_{\mathrm{O}}+q_{\mathrm{He}}^{I} M_{\mathrm{He}}+q_{\mathrm{E}}^{I} M_{\mathrm{E}}}
$$

or

$$
\frac{\left(N_{\mathrm{He}}^{R} / N_{\mathrm{He}}^{I}\right)}{\alpha_{R, I}}=\frac{q_{\mathrm{O}}^{I} M_{\mathrm{O}}}{q_{\mathrm{He}}^{I} M_{\mathrm{He}}}+\frac{q_{\mathrm{E}}^{I} M_{\mathrm{E}}}{q_{\mathrm{He}}^{I} M_{\mathrm{He}}}+1 .
$$

The choice of the stable index isotope used for reference is arbitrary. Let us assume that $M_{\mathrm{O}} \sim M_{\mathrm{SC}}$; and insofar as the envelope is not greatly enhanced in stable nuclei, $q_{\mathrm{E}}^{I} \cong q_{\mathrm{O}}^{I} \cong$ $q_{\mathrm{SC}}^{I}$, we obtain

$$
\frac{M_{\mathrm{SC}}}{M_{\mathrm{He}}}+\frac{M_{\mathrm{E}}}{M_{\mathrm{He}}} \approx\left[\frac{\left(N_{\mathrm{He}}^{R} / N_{\mathrm{He}}^{I}\right)}{\alpha_{R, I}}-1\right] \frac{q_{\mathrm{He}}^{I}}{q_{\mathrm{SC}}^{I}} .
$$

This is a simplified version of the dilution equation in which the ratio $\alpha_{R, I}$ in the cloud is produced by addition of material from the He-burning zone to the ISM. It should be noted that the product $\left(N_{\mathrm{He}}^{R} / N_{\mathrm{He}}^{I}\right) q_{\mathrm{He}}^{I} / q_{\mathrm{SC}}^{I}$ is a determining quantity. The ratio of the mass of $\mathrm{He}$ shell material added to that of the proto-solar cloud is $M_{\mathrm{He}} / M_{\mathrm{Sc}}$. This ratio should be the same for all isotopic species produced by this mechanism. The fraction of stable nuclei that came from this addition of He-shell matter is

$$
\begin{aligned}
\frac{N_{\mathrm{He}}^{I}}{N_{\mathrm{SC}}^{I}} & =\frac{q_{\mathrm{He}}^{I} M_{\mathrm{He}}}{q_{\mathrm{He}}^{I} M_{\mathrm{He}}+q_{\mathrm{O}}^{I} M_{\mathrm{O}}+q_{\mathrm{E}}^{I} M_{\mathrm{E}}} \\
& =\frac{\left(q_{\mathrm{He}}^{I} / q_{\mathrm{O}}^{I}\right)\left(M_{\mathrm{He}} / M_{\mathrm{O}}\right)}{1+\left(q_{\mathrm{He}}^{I} / q_{\mathrm{O}}^{I}\right) M_{\mathrm{He}} / M_{\mathrm{O}}+\left(q_{\mathrm{E}}^{I} / q_{\mathrm{O}}^{I}\right) M_{\mathrm{E}} / M_{\mathrm{O}}} .
\end{aligned}
$$

With the approximations indicated above, and for small additions, equation (4) becomes

$$
\frac{N_{\mathrm{He}}^{I}}{N_{\mathrm{SC}}^{I}} \cong\left(\frac{q_{\mathrm{He}}^{I}}{q_{\mathrm{SC}}^{I}}\right)\left(\frac{M_{\mathrm{He}}}{M_{\mathrm{SC}}}\right) .
$$

In addition to this model of a single injection of freshly processed material, one can consider the continuous addition of AGB matter from many stars at some constant rate $\dot{M}_{\mathrm{He}}$ into the ISM over a prolonged time. We take the molecular cloud (MC) to be well stirred. Let the time over which injection takes place be $t$; in the cloud the total number of stable nuclei produced in the He-burning zone of AGB stars will be

$$
N_{\mathrm{MC}}^{I}(t)=\left(N_{\mathrm{He}}^{I} / M_{\mathrm{He}}\right) \dot{M}_{\mathrm{He}} t+N_{\mathrm{MC}}^{I}(0) .
$$

If the fraction of all the stable nuclei $I$ that are produced by the continuous AGB injection in the cloud is $f_{I}$, then

$f_{I} \equiv \frac{N_{\mathrm{MC}}^{I}(t)-N_{\mathrm{MC}}^{I}(O)}{N_{\mathrm{MC}}^{I}(t)}=\left(\frac{N_{\mathrm{He}}^{I}}{M_{\mathrm{He}}}\right) \frac{\dot{M}_{\mathrm{He}} t}{N_{\mathrm{MC}}^{I}(t)}=\frac{q_{\mathrm{He}}^{I}}{q_{\mathrm{MC}}^{I}} \frac{\dot{M}_{\mathrm{He}}}{M_{\mathrm{MC}}} t$.

For a radioactive nuclide with mean life $\bar{\tau}_{R}$ much shorter than $t$ :

$$
N_{\mathrm{MC}}^{R}=\left(\frac{N_{\mathrm{He}}^{R}}{M_{\mathrm{He}}}\right) \dot{M}_{\mathrm{He}} \bar{\tau}_{R} .
$$

It follows that the relative abundance of the radioactive nucleus to the stable one in the cloud is given by

$$
\left(\frac{N_{\mathrm{MC}}^{R}}{N_{\mathrm{MC}}^{I}}\right)=\left(\frac{N_{\mathrm{He}}^{R}}{N_{\mathrm{He}}^{I}}\right)\left(\frac{q_{\mathrm{He}}^{I}}{q_{\mathrm{MC}}^{I}}\right)\left(\frac{\dot{M}_{\mathrm{He}}}{M_{\mathrm{MC}}}\right) \bar{\tau}_{R} .
$$

Using equation (9) we can calculate the corresponding value of $\left(\dot{M}_{\mathrm{He}} / M_{\mathrm{MC}}\right)$ if the ratios in the cloud and the He shell are known for a pair of nuclei. Using this value and assuming a time $t$ one may calculate $f_{I}$ for arbitrary species from equation (7). Equation (9) is similar to the results given by Schramm \& Wasserburg (1970). The constant rates given here can obviously be replaced with the average rates for nuclei $R$ and $I$ over the respective time periods, $\bar{\tau}_{R}$ and $t$. Again, for a self-consistent model, the ratio $\dot{M}_{\mathrm{He}} / M_{\mathrm{MC}}$ should be the same for all isotopic species produced by this mechanism.

\section{RESULTS FOR SHORT-LIVED ISOTOPES}

Here we apply the models described above to the various radioactive nuclei shown in Table 1 . The values given are for the He shell in AGB stars in the range of 1.5 to $3 M_{\odot}$. From the results on stellar models exhibited in Figures 3 to 6, we obtain the relative abundances $N_{\mathrm{He}}^{R} / N_{\mathrm{He}}^{I}$ of unstable $(R)$ and stable $(I)$ isotopes in the He-shell matter. Starting with the case of prompt contamination by a single AGB star, we first analyze an $s$-process nucleus (e.g., ${ }^{107} \mathrm{Pd}$ ) for which a reliable measured abundance at the epoch of the solar system formation exists. For this nucleus we derive the dilution factors to be applied to the He-shell concentrations to explain the experimental abundances. Then, assuming ${ }^{107} \mathrm{Pd}$ as a reference, we apply the dilution factor deduced for it to all the other short-lived nuclei that can receive contributions from the $s$-process starting with those nuclei which lie on the main $s$-process path $\left({ }^{99} \mathrm{Tc},{ }^{93} \mathrm{Zr}\right.$, ${ }^{205} \mathrm{~Pb}$ ) and then consider those that critically depend on branching points in the s-chain $\left({ }^{60} \mathrm{Fe},{ }^{135} \mathrm{Cs},{ }^{182} \mathrm{Hf}\right)$. From the nuclei having good abundance estimates, we derive further constraints on the model parameters. For the other nuclei, we give model estimates of the early solar nebular abundances that can be tested by future experimental work. An estimate is also given for the small AGB contribution to ${ }^{129} \mathrm{I}$. The measured abundance of ${ }^{26} \mathrm{Al}$ is then independently used to compute the dilution factor for the $\mathrm{H}$ shell product $\left(M_{\mathrm{H}} / M_{\mathrm{SC}}\right)$. We then look for a possible self-consistent interpretation, valid both for He-shell and for $\mathrm{H}$-shell products. Finally the whole analysis is done for the continuous addition scenario.

\subsection{Radioactive Nuclei not Involving Branching}

The Case of ${ }^{107} \mathrm{Pd}$.- For this nucleus, there is clear evidence of its existence in the early solar system, with a ratio ${ }^{107} \mathrm{Pd} /$ ${ }^{108} \mathrm{Pd} \sim 2 \times 10^{-5}$ (Kelly \& Wasserburg 1978; Kaiser \& Was- 
TABLE 2

INSTANT INJECTION AND MIXINGa

\begin{tabular}{lllll}
\hline \hline \multicolumn{1}{c}{ Isotope Ratio } & $N_{\mathrm{He}}^{R} / N_{\mathrm{He}}^{I}$ & $q_{\mathrm{He}}^{I} / q_{\mathrm{SC}}^{I}$ & $\begin{array}{c}\alpha_{R, I} \\
\text { (Predicted) }\end{array}$ & $\begin{array}{c}\alpha_{R, I} \\
\text { (Observed) }\end{array}$ \\
\hline${ }^{107} \mathrm{Pd} /{ }^{108} \mathrm{Pd} \ldots \ldots \ldots \ldots$ & 0.184 & 1.28 & $2 \times 10^{-5}$ & $2 \times 10^{-5}$ \\
${ }^{205} \mathrm{~Pb} /{ }^{204} \mathrm{~Pb} \ldots \ldots \ldots \ldots$ & 1.39 & 3.40 & $4.0 \times 10^{-4}$ & \\
${ }^{93} \mathrm{Zr} /{ }^{92} \mathrm{Zr} \ldots \ldots \ldots \ldots \ldots$ & 0.237 & 1.26 & $2.5 \times 10^{-5}$ & \\
${ }^{99} \mathrm{Tc} /{ }^{100} \mathrm{Ru} \ldots \ldots \ldots \ldots \ldots$ & 0.161 & 1.68 & $2.3 \times 10^{-5}$ & \\
${ }^{60} \mathrm{Fe} /{ }^{56} \mathrm{Fe} \ldots \ldots \ldots \ldots \ldots$ & $1.0 \times 10^{-4}$ & 0.854 & $7.3 \times 10^{-9}$ & $4 \times 10^{-9}$ \\
${ }^{135} \mathrm{Cs} /{ }^{133} \mathrm{Cs} \ldots \ldots \ldots \ldots$ & 0.448 & 0.734 & $2.8 \times 10^{-5}$ & \\
${ }^{182} \mathrm{Hf} /{ }^{180} \mathrm{Hf} \ldots \ldots \ldots \ldots$ & 0.0116 & 2.64 & $2.6 \times 10^{-6}$ & \\
${ }^{129} \mathrm{I} /{ }^{127} \mathrm{I} \ldots \ldots \ldots \ldots \ldots$ & 0.0106 & 0.200 & $1.8 \times 10^{-7}$ & $1 \times 10^{-4}$ \\
${ }^{26} \mathrm{Al} /{ }^{27} \mathrm{Al} \ldots \ldots \ldots \ldots \ldots$ & $0.8 \mathrm{~b}$ & 1.0 & $4.8 \times 10^{-5}-1.3 \times 10^{-4 \mathrm{c}}$ & $5 \times 10^{-5}$ \\
\hline
\end{tabular}

a $\tau_{0}=0.03 \mathrm{mbarn}^{-1} ; n_{n}^{\mathrm{Max}}=2.1 \times 10^{8} \mathrm{~cm}^{-3} ; M_{\mathrm{He}} / M_{\mathrm{SC}}=8.5 \times 10^{-5}$.

b Number ratio in the $\mathrm{H}$-shell.

c This range of $\alpha$ is deduced applying the ratio of $M_{\mathrm{H}} / M_{\mathrm{He}}=(0.7-2)$ discussed in the text.

serburg 1983; Chen \& Wasserburg 1983, 1990; cf. Wasserburg 1985). All of the Pd isotopes lie on the main $s$-process chain, i.e., the flow leading to ${ }^{107} \mathrm{Pd}$ is unbranched. Due to this fact, and to the short duration of the neutron fluences (of the order of $10 \mathrm{yr}$ ) as compared to its mean life, slow neutron captures are a dominant mechanism for production of ${ }^{107} \mathrm{Pd}$, though it can also receive contributions from the $r$-process, since ${ }^{107} \mathrm{Pd}$ is not shielded by stable isobars. Also for stable ${ }^{108} \mathrm{Pd}$, the contribution from the $s$-process is consistent, amounting to $66 \%$ of the total abundance, as can be derived from the data of Gallino et al. (1993). The values of $N_{\mathrm{He}}^{107} / N_{\mathrm{He}}^{108}$ and $q_{\mathrm{He}}^{108} / q_{\mathrm{SC}}^{108}$ for the four cases of Figures 3 and 6 are presented in Tables 2 to 5 . Inspection of these tables shows the enrichment factor $q_{\mathrm{He}}^{108} / q_{\mathrm{SC}}^{108}$ to be a very sensitive function of $\tau_{0}$, ranging from $1.28\left(\tau_{0}=0.03\right.$ mbarn $\left.^{-1}\right)$ to $891\left(\tau_{0}=0.28\right.$ mbarn $\left.^{-1}\right)$. In contrast, the ratio $N_{\mathrm{He}}^{107} / N_{\mathrm{He}}^{108}$ is almost independent of $\tau_{0}$, due to the fact that here the $s$-chain is unbranched and the cross sections of all Pd isotopes are fairly large. The constancy of $N_{\mathrm{He}}^{107} / N_{\mathrm{He}}^{108}$ is in general agreement with the study by Cameron (1993). Since both the production parameters and the abundances in the early solar system are well established and the lifetime of radioactive ${ }^{107} \mathrm{Pd}$ is rather long, we adopt this isotropic pair as a reference. We assume that the mass of the envelope ejected from the star is about 50 times the mass of the He-shell that is dredged up as this is the result we typically find in the mixing procedure leading to carbon star formation. From the parameters given in Tables 2 to 5 and using $M_{\mathrm{E}} / M_{\mathrm{He}} \sim 50$ we obtain from equation (3): $M_{\mathrm{He}} / M_{\mathrm{SC}}=8.5$ $\times 10^{-5}, 2.36 \times 10^{-5}, 1.7 \times 10^{-6}$, and $1.4 \times 10^{-7}$ in the four cases studied. The prompt mixing of these small amounts of He-burning matter into $1 M_{\odot}$ of a pre-solar cloud is sufficient to readily account for the abundance of ${ }^{107} \mathrm{Pd}$ in the early solar nebula. The ratio of freshly synthesized ${ }^{108} \mathrm{Pd}$ to that already present in the cloud is $\sim 10^{-4}$ (see eq. [5]).

${ }^{205} \mathrm{~Pb}$.- This is a crucial nuclide, as it is shielded from the $r$-process and can receive contributions only in slow neutron capture environments; the same is true for the stable isotope ${ }^{204} \mathrm{~Pb}$ used as a reference (Blake, Lee, \& Schramm 1973). An incisive review of the nucleosynthesis of ${ }^{205} \mathrm{~Pb}$ with consideration of the $\beta^{-}$decay of ${ }^{205} \mathrm{Tl}$, and of the lifetime of ${ }^{205} \mathrm{~Pb}$ in astrophysical conditions, is given in Yokoi, Takahashi, \& Arnould (1985). They have emphasized that the production ratio of ${ }^{205} \mathrm{~Pb} /{ }^{204} \mathrm{~Pb}$, ranging from 0.4 to 1.7 for a parametric neutron irradiation, is high enough to warrant a renewed search for ${ }^{205} \mathrm{~Pb}$ in the early solar system. Our calculations of $N_{\mathrm{He}}^{205} / N_{\mathrm{He}}^{204}$, as given in Tables 2-5 for the four values of $\tau_{0}$ adopted, essentially confirm the resullts by the above mentioned authors; we also estimate, for the same cases, $q_{\mathrm{He}}^{204} / q_{\mathrm{SC}}^{204}$ ranging from 3.40 to 1083 . The calculated production is dependent on the cross section of ${ }^{205} \mathrm{~Pb}$ which is uncertain by at least

TABLE 3

INSTANT INJECTION AND MIXINGa

\begin{tabular}{llccc}
\hline \hline \multicolumn{1}{c}{ Isotope Ratio } & \multicolumn{1}{c}{$N_{\mathrm{He}}^{R} / N_{\mathrm{He}}^{I}$} & $q_{\mathrm{He}}^{I} / q_{\mathrm{SC}}^{I}$ & $\begin{array}{c}\alpha_{R, I} \\
\text { (Predicted) }\end{array}$ & $\begin{array}{c}\alpha_{R, I} \\
\text { (Observed) }\end{array}$ \\
\hline${ }^{107} \mathrm{Pd} /{ }^{108} \mathrm{Pd} \ldots \ldots \ldots \ldots$ & 0.174 & 4.87 & $2 \times 10^{-5}$ & $2 \times 10^{-5}$ \\
${ }^{205} \mathrm{~Pb} /{ }^{204} \mathrm{~Pb} \ldots \ldots \ldots \ldots$ & 1.55 & 4.27 & $1.6 \times 10^{-4}$ & \\
${ }^{93} \mathrm{Zr} /{ }^{2} \mathrm{Zr} \ldots \ldots \ldots \ldots \ldots$ & 0.309 & 11.87 & $8.7 \times 10^{-5}$ & \\
${ }^{99} \mathrm{Tc} /{ }^{100} \mathrm{Ru} \ldots \ldots \ldots \ldots$ & 0.181 & 9.1 & $3.9 \times 10^{-5}$ & \\
${ }^{60} \mathrm{Fe} /{ }^{56} \mathrm{Fe} \ldots \ldots \ldots \ldots \ldots$ & $3.7 \times 10^{-4}$ & 0.74 & $6.5 \times 10^{-9}$ & $4 \times 10^{-9}$ \\
${ }^{135} \mathrm{Cs} /{ }^{133} \mathrm{Cs} \ldots \ldots \ldots \ldots$ & 0.429 & 0.89 & $9.0 \times 10^{-6}$ & \\
${ }^{18} \mathrm{Hf} /{ }^{180} \mathrm{Hf} \ldots \ldots \ldots \ldots$ & 0.0123 & 2.43 & $7.5 \times 10^{-7}$ & \\
${ }^{129} \mathrm{I} /{ }^{127} \mathrm{I} \ldots \ldots \ldots \ldots \ldots$ & $5.84 \times 10^{-3}$ & 0.34 & $4.8 \times 10^{-8}$ & $1 \times 10^{-4}$ \\
${ }^{26} \mathrm{Al} /{ }^{27} \mathrm{Al} \ldots \ldots \ldots \ldots$. & $0.8^{\mathrm{b}}$ & 1.0 & $1.3 \times 10^{-5}-3.8 \times 10^{-5 \mathrm{c}}$ & $5 \times 10^{-5}$ \\
\hline
\end{tabular}

a $\tau_{0}=0.07 \mathrm{mbarn}^{-1} ; n_{n}^{\mathrm{Max}}=2.0 \times 10^{8} \mathrm{~cm}^{-3} ; M_{\mathrm{He}} / M_{\mathrm{SC}}=2.4 \times 10^{-5}$.

${ }^{b}$ Number ratio in the $\mathrm{H}$-shell.

c This range of $\alpha$ is deduced applying the ratio of $M_{\mathrm{H}} / M_{\mathrm{He}}=(0.7-2)$ discussed in the text. 
TABLE 4

INSTANT INJECTION AND MIXING ${ }^{a}$

\begin{tabular}{llccc}
\hline \hline \multicolumn{1}{c}{ Isotope Ratio } & \multicolumn{1}{c}{$N_{\mathrm{He}}^{R} / N_{\mathrm{He}}^{I}$} & $q_{\mathrm{He}}^{I} / q_{\mathrm{SC}}^{I}$ & $\begin{array}{c}\alpha_{R, I} \\
\text { (Predicted) }\end{array}$ & $\begin{array}{c}\alpha_{R, I} \\
\text { (Observed) }\end{array}$ \\
\hline${ }^{107} \mathrm{Pd} /{ }^{108} \mathrm{Pd} \ldots \ldots \ldots \ldots$ & 0.163 & 72.4 & $2 \times 10^{-5}$ & $2 \times 10^{-5}$ \\
${ }^{205} \mathrm{~Pb} /{ }^{204} \mathrm{~Pb} \ldots \ldots \ldots \ldots$ & 1.66 & 13.9 & $3.9 \times 10^{-5}$ & \\
${ }^{93} \mathrm{Zr} /{ }^{92} \mathrm{Zr} \ldots \ldots \ldots \ldots \ldots$ & 0.33 & 134 & $7.5 \times 10^{-5}$ & \\
${ }^{99} \mathrm{Tc} /{ }^{100} \mathrm{Ru} \ldots \ldots \ldots \ldots$ & 0.18 & 128 & $3.9 \times 10^{-5}$ & \\
${ }^{60} \mathrm{Fe} /{ }^{56} \mathrm{Fe} \ldots \ldots \ldots \ldots$. & $6.6 \times 10^{-4}$ & 0.653 & $7.3 \times 10^{-10}$ & $4 \times 10^{-9}$ \\
${ }^{135} \mathrm{Cs} /{ }^{133} \mathrm{Cs} \ldots \ldots \ldots \ldots$ & 0.445 & 11.1 & $8.8 \times 10^{-6}$ & \\
${ }^{182} \mathrm{Hf} /{ }^{180} \mathrm{Hf} \ldots \ldots \ldots \ldots$ & 0.0115 & 14.8 & $2.9 \times 10^{-7}$ & \\
${ }^{129} \mathrm{I} /{ }^{127} \mathrm{I} \ldots \ldots \ldots \ldots \ldots$. & $2.9 \times 10^{-3}$ & 4.49 & $2.2 \times 10^{8}$ & $1.0 \times 10^{-4}$ \\
${ }^{26} \mathrm{Al} /{ }^{17} \mathrm{Al} \ldots \ldots \ldots \ldots$. & $0.8^{\mathrm{b}}$ & 1.0 & $9.6 \times 10^{-7}-2.7 \times 10^{-6 \mathrm{c}}$ & $5 \times 10^{-5}$ \\
\hline
\end{tabular}

$\tau_{0}=0.13 \mathrm{mbarn}^{-1} ; n_{n}^{\mathrm{Max}}=1.8 \times 10^{8} \mathrm{~cm}^{-3} ; M_{\mathrm{He}} / M_{\mathrm{SC}}=1.7 \times 10^{-6}$.

b Number ratio in the $\mathbf{H}$-shell.

c This range of $\alpha$ is deduced applying the ratio of $M_{\mathrm{H}} / M_{\mathrm{He}}=(0.7-2)$ discussed in the text.

$20 \%$. A much higher uncertainty derives from the problem of ${ }^{205} \mathrm{~Pb}$ survival during the interpulse phases as stressed by Yokoi et al. (1985). The abundance of ${ }^{205} \mathrm{~Pb}$ is dependent on the $\beta^{-}$decay of ${ }^{205} \mathrm{Tl}$ and the decay of ${ }^{205} \mathrm{~Pb}$ by electron capture, which is a strong function of electron density. Soon after the pulse, due to stellar expansion, the region which will subsequently be mixed to the envelope (region B in Fig. 1) experiences quite low values of temperature and electron density $\left(T \leq 2 \times 10^{7} \mathrm{~K}, n_{e} \sim 10^{25} e \mathrm{~cm}^{-3}\right)$. This stage lasts for about 300 years before convective penetration from the envelope reaches its maximum extension. The quoted local conditions are in a range of parameters where the rate of ${ }^{205} \mathrm{~Pb}$ decay to ${ }^{205} \mathrm{Tl}$ is steeply varying, from about $\sim 1 \mathrm{~s}^{-1}$ (at $T \sim 1.5 \times 10^{8}, n_{e} \sim 10^{26} e^{-} \mathrm{cm}^{-3}$ ) down to $10^{-15} \mathrm{~s}^{-1}$ (the laboratory value). Inspection and extrapolation of the data by Yokoi et al. (1985) would tentatively suggest that the correct rate could be of the order of a few $10^{-10} \mathrm{~s}^{-1}$; however, this extrapolation is in a region where the rate variation covers several orders of magnitude and is not safe. In our calculations we simply assumed the terrestrial lifetime for this pre-mixing phase. Hence, our predictions for ${ }^{205} \mathrm{~Pb}$ are to be seen only as upper limits. Obviously, a precise evaluation of the $e^{-}$capture rate of ${ }^{205} \mathrm{~Pb}$ over the interpulse evolution is required for a real evaluation. To give an idea of the uncertainty, if we had adopted the above mentioned extrapolation of a few $10^{-10} \mathrm{~s}^{-1}$ instead of the laboratory value for the rate of ${ }^{205} \mathrm{~Pb}$ decay before dredge-up, our estimates for $\alpha_{205,204}$ would be reduced by at least a factor of 10 . Using the dilution factors for ${ }^{107} \mathrm{Pd}$ for the respective cases and taking the terrestrial lifetime of ${ }^{205} \mathrm{~Pb}$ for the interpulse phase, we obtain $\alpha_{205,204}$ in the narrow range $0.4-4.0 \times 10^{-4}$. Ostic, El-Badry, \& Kohman, (1969), and Huey \& Kohman (1972) reported an upper limit of $\left({ }^{205} \mathrm{~Pb} /{ }^{204} \mathrm{~Pb}\right) \sim 9 \times 10^{-5}$ for some meteorites. These experimental upper bounds are not precise but appear to be essentially compatible with the upper bounds obtained here. An extensive discussion of these issues will be given in $\S 5$.

${ }^{93} \mathrm{Zr}$. - This nuclide lies on the main $s$-process chain. For all $\mathrm{Zr}$ isotopes there are some contributions from the $r$-process, but as discussed by Käppeler et al. 1990, the dominant role is played by slow neutron captures. In particular, these are responsible for about $83 \%$ of solar $\mathrm{Nb}$ from the ${ }^{93} \mathrm{Zr}$ decay. Observations of the isotopic composition of $\mathrm{Zr}$ were derived spectroscopically for some MS, S, and C stars by Zook (1985) and by Lambert (1991), while the presence of its decay product $\mathrm{Nb}$ was reported by Dominy \& Wallerstein (1986). Despite the difficulty of these observations and their high uncertainty, they appear to confirm the production of ${ }^{93} \mathrm{Zr}$ in AGB stars. With regard to identification of ${ }^{93} \mathrm{Zr}$ in solar system material, as $\mathrm{Nb}$ has only one stable isotope, no direct measurement of ${ }^{93} \mathrm{Zr}$ decay is possible. We may calculate the abundance of this

TABLE 5

INSTANT INJECTION AND MIXING ${ }^{\mathrm{a}}$

\begin{tabular}{|c|c|c|c|c|}
\hline Isotope Ratio & $N_{\mathrm{He}}^{R} / N_{\mathrm{He}}^{I}$ & $q_{\mathrm{He}}^{I} /={ }_{\mathrm{SC}}^{I}$ & $\begin{array}{c}\alpha_{R, I} \\
\text { (Predicted) }\end{array}$ & $\begin{array}{c}\alpha_{R, I} \\
\text { (Observed) }\end{array}$ \\
\hline${ }^{107} \mathrm{Pd} /{ }^{108} \mathrm{Pd} \ldots \ldots \ldots \ldots$ & 0.158 & 891 & $2 \times 10^{-5}$ & $2 \times 10^{-5}$ \\
\hline${ }^{205} \mathrm{~Pb} /{ }^{204} \mathrm{~Pb} \ldots \ldots \ldots \ldots$ & 1.83 & 1083 & $2.8 \times 10^{-4}$ & \\
\hline${ }^{93} \mathrm{Zr} /{ }^{92} \mathrm{Zr} \ldots \ldots \ldots \ldots \ldots$ & 0.334 & 1158 & $5.6 \times 10^{-5}$ & \\
\hline${ }^{99} \mathrm{Tc} /{ }^{100} \mathrm{Ru} \ldots \ldots \ldots \ldots$ & 0.176 & 1461 & $3.6 \times 10^{-5}$ & \\
\hline${ }^{60} \mathrm{Fe} /{ }^{56} \mathrm{Fe} \ldots \ldots \ldots \ldots \ldots$ & $2.56 \times 10^{-3}$ & 0.520 & $1.8 \times 10^{-10}$ & $4 \times 10^{-9}$ \\
\hline${ }^{135} \mathrm{Cs} /{ }^{133} \mathrm{Cs} \ldots \ldots \ldots \ldots$ & 0.712 & 222 & $2.2 \times 10^{-5}$ & \\
\hline${ }^{182} \mathrm{Hf} /{ }^{180} \mathrm{Hf}$........... & 0.0241 & 1030 & $3.5 \times 10^{-6}$ & \\
\hline${ }^{129} \mathrm{I} /{ }^{127} \mathrm{I} \ldots \ldots$ & $2.58 \times 10^{-3}$ & 79.3 & $5.7 \times 10^{-8}$ & $1.0 \times 10^{-4}$ \\
\hline${ }^{26} \mathrm{Al} /{ }^{27} \mathrm{Al} \ldots$ & $0.8^{\mathrm{b}}$ & 1.0 & $8.06 \times 10^{-8}-2.3 \times 10^{-7 c}$ & $5 \times 10^{-5}$ \\
\hline
\end{tabular}


nucleus in the prompt contamination model presented here assuming the same dilution obtained for ${ }^{107} \mathrm{Pd}$. For the cases of Tables 2-5, the expected values of $\alpha_{93,92}$ range from $2.5 \times 10^{-5}$ to $8.7 \times 10^{-5}$

${ }^{99} \mathrm{Tc}$. - While this nuclide has a mean life much shorter than $10^{6} \mathrm{yr}$, we have included it because it was observed in stellar spectra over a long period of time (Merrill 1952; for recent data see Smith \& Lambert 1988 and Kipper 1991). The s-chain passes through ${ }^{98} \mathrm{Mo}$ and ${ }^{99} \mathrm{Mo}$, which mainly decays to ${ }^{99} \mathrm{Tc}$. The flow is then through ${ }^{99} \mathrm{Tc}$ to the very short-lived ${ }^{100} \mathrm{Tc}$, to produce ${ }^{100} \mathrm{Ru}$. Some leakage from the $s$-flow also occurs directly from ${ }^{99} \mathrm{Tc}$ to ${ }^{99} \mathrm{Ru}$, accounting for about $28 \%$ of ${ }^{99} \mathrm{Ru}$ abundance from the $s$-process. This is mainly due to the interpulse decay of Tc and (in minor part) to its enhanced decay $(\bar{\tau}=6.5 \mathrm{yr})$ in the last stages of the pulse, where the average temperature reaches $26 \mathrm{keV}$. As there are no stable Tc isotopes, the index isotope used is ${ }^{100} \mathrm{Ru}$. If we neglect for a moment the radioactive decay in the envelope and in the ISM (i.e., assuming a timescale as short as $10^{5} \mathrm{yr}$ for mixing into the solar nebula) and follow the calculation used for ${ }^{107} \mathrm{Pd}$, we obtain $\alpha_{99,100} \approx 3.4 \times 10^{-5}$ for all $\tau_{0}$ values. If the material of the solar system collected very rapidly, then samples that formed with ratios $T c / R u \simeq 10$ would yield shifts of ${ }^{99} \mathrm{Ru}$ / ${ }^{100} \mathrm{Ru}$ of 10 parts over $10^{4}$ (i.e., 10єu). For an aggregation time of $\simeq 10^{6} \mathrm{yr}$, this effect would of course by reduced by a factor of 30 .

\subsection{Radioactive Nuclei Depending on Branching}

${ }^{60} \mathrm{Fe}$.- Unambiguous evidence for the presence of ${ }^{60} \mathrm{Fe}$ has recently been discovered in differentiated planetary material by Shukolyukov \& Lugmair (1993) with the abundance of ${ }^{60} \mathrm{Fe} /$ ${ }^{56} \mathrm{Fe} \approx 4 \times 10^{-9}$. Though ${ }^{56} \mathrm{Fe}$ is the main seed for all the $s$-process, in our calculations its abundance at the end of a pulse is not negligible, being reduced only to between $86 \%$ and $52 \%$ of the initial value in the models considered (see Tables $2-5$ ). These rather high concentrations of ${ }^{56} \mathrm{Fe}$ in the He zone can be understood in light of the complex evolution of the convective instability model; indeed, while ${ }^{56} \mathrm{Fe}$ is strongly depleted by neutron captures during the activation of the ${ }^{13} \mathrm{C}$ neutron source in the first phase of each pulse, its abundance is subsequently partly restored by ingestion of unprocessed material when convection expands in the final stages. The neutron capture flow produces ${ }^{59} \mathrm{Fe}$ which mainly decays to ${ }^{59} \mathrm{Co}$, with a lifetime $\bar{\tau}=65.4$ days which is independent of temperature. However, a minor channel of neutron captures on ${ }^{59} \mathrm{Fe}$ also occurs, at a level sufficient to produce some ${ }^{60} \mathrm{Fe}$ (see Fig. 7). The resulting values of $N_{\mathrm{He}}^{60} / N_{\mathrm{He}}^{56}$ are from $1.0 \times 10^{-4}$ to $2.6 \times 10^{-3}$, for the cases of Tables $2,3,4$, and 5 . The ratios of ${ }^{60} \mathrm{Fe} /{ }^{56} \mathrm{Fe}$ are quite small, and have $q_{\mathrm{He}}^{56} / q_{\mathrm{SC}}^{56} \sim 1$. Nonetheless, there is a most substantial contribution of ${ }^{60} \mathrm{Fe}$ to the solar nebular cloud. Using the values of $M_{\mathrm{He}} / M_{\mathrm{SC}}$ obtained for ${ }^{107} \mathrm{Pd} /{ }^{108} \mathrm{Pd}$ for the various cases, we obtain $\alpha_{60,56}$ ranging from $7.3 \times 10^{-9}$ at $\tau_{0}=0.03 \mathrm{mbarn}^{-1}$ to $1.8 \times 10^{-10}$ at $\tau_{0}=$ $0.28 \mathrm{mbarn}^{-1}$. One notes that for both ${ }^{59} \mathrm{Fe}$ and ${ }^{60} \mathrm{Fe}$, only theoretical estimates for the neutron capture cross sections are available (12 and 3.6 mbarn, respectively, as given by Woosley et al. 1978). Typical uncertainties of these estimates are of a factor of 2 , and we notice that abundances of ${ }^{60} \mathrm{Fe}$ given above would scale proportionately with the cross section of ${ }^{59} \mathrm{Fe}$. It follows that AGB stars may provide significant sources of ${ }^{60} \mathrm{Fe}$ in the ISM and to the proto-solar cloud in particular. As can be seen from Tables 2 and 3, the cases of $\tau_{0}=0.03$ and 0.07 mbarn $^{-1}$ are sufficient to obtain the value of $4 \times 10^{-9}$ found

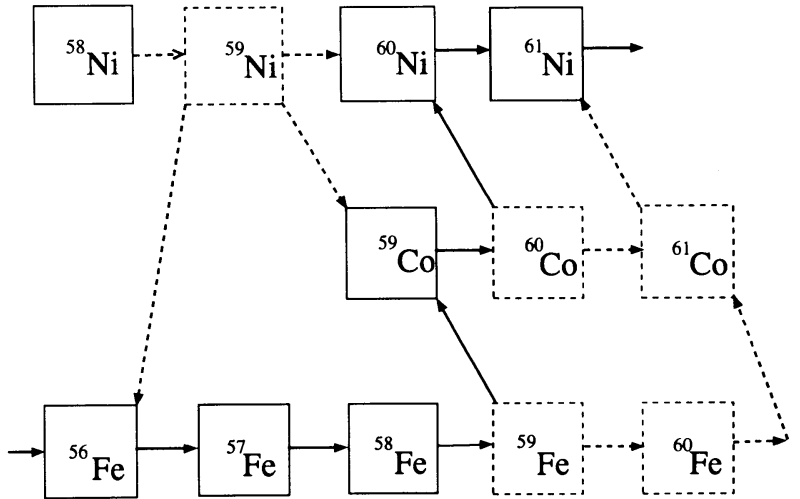

Fig. 7.-Zone of the neutron capture path involving ${ }^{60} \mathrm{Fe}$. Notice that, due to the short duration of the neutron pulses in the $s$-flow ${ }^{60} \mathrm{Fe}$ behaves practically as a stable nucleus.

in a meteorite by Shukolyukov \& Lugmair (1993). The question of the abundance of ${ }^{60} \mathrm{Fe}$ in this planetary object as compared to the proto-solar cloud depends on the time interval, $\Delta$, between the time of injection and the formation of the planetary differentiate as pointed out by these workers. This matter will be discussed in $\S 5$ along with other pertinent nuclides.

${ }^{135} \mathrm{Cs}$. - The stable nucleus ${ }^{133} \mathrm{Cs}$ lies on the main $s$-path but, owing to its fairly large neutron capture cross section, it receives only about a $16 \%$ contribution by the main component; the remaining $84 \%$ is to be ascribed to the $r$-process. For a full discussion of the uncertainties of nuclear parameters in this region see Gallino et al. (1993). The $s$-flow branches at ${ }^{134} \mathrm{Cs}$ : this isotope has a terrestrial mean life of $3 \mathrm{yr}$, but the lifetime is considerably reduced (by a factor of 10) in typical thermal pulse conditions. A small neutron capture channel feeds ${ }^{135} \mathrm{Cs}$, which has $\bar{\tau}=3 \times 10^{6}$ yr under laboratory conditions. In He-shell conditions, this lifetime is also strongly reduced (see Table 1), but, in any case, it remains longer than the duration of the $s$-fluence. The third dredge-up operates quite soon after the quenching of the thermal instability and the ${ }^{135} \mathrm{Cs}$ nuclei brought into the cool envelope will decay there with the terrestrial mean life. Using the same dilution calculation used above we find $\alpha_{135 / 133}$ ranging from $9 \times 10^{-6}$, to $2.8 \times 10^{-5}$. Cameron et al. (1993) showed that the abundance of ${ }^{135} \mathrm{Cs}$ steadily increases with increasing values of $n_{n}$. However, we do not find any substantial regular increases of ${ }^{135} \mathrm{Cs} /{ }^{133} \mathrm{Cs}$ in our model, nor do we find it greatly sensitive to $n_{n}$ (see end of this section). This apparent inconsistency can be easily explained. Indeed, in our model, ${ }^{135} \mathrm{Cs}$ is produced mainly in the low-temperature phase of the pulse, where ${ }^{13} \mathrm{C}$ is burnt (phase 2 in Fig. 2). Here,${ }^{134} \mathrm{Cs}$ has a lifetime of $10^{7} \mathrm{~s}$ and, due to its large cross section (1 barn), neutron captures on it leading to ${ }^{135} \mathrm{Cs}$ are always an important reaction channel, accounting for about $35 \%$ of the flow in standard conditions $\left(n_{n}=2 \times 10^{8} n \mathrm{~cm}^{-3}\right)$. Hence, even when $n_{n}$ is increased by orders of magnitude, ${ }^{135} \mathrm{Cs}$ production cannot vary by more than a factor of 3 (i.e., up to $100 \%$ of the flow at ${ }^{134} \mathrm{Cs}$ ). By contrast, in a parametric study at the constant higher temperature of $30 \mathrm{keV}$, as in Cameron et al. (1993), the decay of ${ }^{134} \mathrm{Cs}$ to ${ }^{134} \mathrm{Ba}$ is favored (the lifetime becomes of the order of $3 \times 10^{5} \mathrm{~s}$ ), so that the production of ${ }^{135} \mathrm{Cs}$ becomes marginal ( $1 \%$ to $2 \%$ at $\left.n_{n}=2 \times 10^{9} n \mathrm{~cm}^{-3}\right)$, its abundance varying almost linearly with the neutron density. The evaluation of ${ }^{135} \mathrm{Cs}$ abundance in the early solar system would, therefore, 
yield an important constraint on the average temperature in the He-shell during neutron captures.

${ }^{182} \mathrm{Hf}$. - The main $s$-process component produces about $60 \%$ of solar ${ }^{180} \mathrm{Hf}$ and about $47 \%$ of solar ${ }^{182} \mathrm{~W}$ (Gallino et al. 1993). The $s$-process neutron capture path proceeds predominantly through ${ }^{181} \mathrm{Hf}$ and to ${ }^{181} \mathrm{Ta}$; at ${ }^{181} \mathrm{Hf}$ there is a minor branching to ${ }^{182} \mathrm{Hf}$. The isotope ${ }^{181} \mathrm{Hf}$ has a laboratory mean life $\bar{\tau}=62.4$ days, that is reduced by about a factor of 30 at $T=3 \times 10^{8} \mathrm{~K}$. The predicted ${ }^{182} \mathrm{Hf}$ abundance depends on the neutron density experienced by the $s$-process environment, but very little on the temperature, since under astrophysical conditions its mean life remains longer by at least a factor of 10 than the duration of the neutron flux, and in the envelope ${ }^{182} \mathrm{Hf}$ has its terrestrial half-life. Using the scheme outlined above we get predictions of $\alpha_{182,180}$ from $2.9 \times 10^{-7}$ to $3.5 \times 10^{-6}$. The highest value is obtained in the model with $\tau_{0}=0.28$ mbarn $^{-1}$, which has the highest $n_{n}$ value. Since the production ratio ${ }^{182} \mathrm{Hf} /{ }^{182} \mathrm{~W}$ is small, in $s$-process conditions this last nucleus derives mainly from the flow through ${ }^{181} \mathrm{Ta}$ and only marginally from ${ }^{182} \mathrm{Hf}$ decay. We note that to observe a shift of $1 \epsilon u$ in ${ }^{182} \mathrm{~W}$ from ${ }^{182} \mathrm{Hf}$ decay would require a sample to have a ratio $\mathrm{Hf} / \mathrm{W}$ of 142,200 , or 17 , for the three largest values of $\tau_{0}$.

${ }^{53} \mathrm{Mn}$.- The production of ${ }^{53} \mathrm{Mn}$ remains problematic. In particular, in AGB stars there are no channels feeding ${ }^{53} \mathrm{Mn}$ during the $s$-fluence in the $\mathrm{He}$ shell; it can only receive contributions from proton captures on ${ }^{52} \mathrm{Cr}$ in the $\mathrm{H}$ shell. However, due to the extremely low value of the reaction rate in these conditions, its production is several orders of magnitude below what is required. This isotope can be produced efficiently in nuclear statistical equilibrium and could be accounted for by the continuous injection of supernovae (Type I) material into the ISM (Cameron 1993). The short lifetime ( $\left.\bar{\tau}=5.3 \times 10^{6} \mathrm{yr}\right)$ may pose a difficulty for continuous injection. Another possible source of ${ }^{53} \mathrm{Mn}$ could be charged particle bombardment of dust in the early solar system, by an early $T$ Tauri phase of the Sun (cf. Clayton, Dwek, \& Woosley 1977); however, this must take place after the gas was blown away. In a study by Wasserburg \& Arnould (1987), it was shown that ${ }^{53} \mathrm{Mn}$ is produced by energetic particle reactions on dust far more efficiently than ${ }^{26} \mathrm{Al}$. The details depend on the nature of the particle spectrum (power or exponential). However, it is possible to obtain ${ }^{53} \mathrm{Mn} /{ }^{55} \mathrm{Mn} \sim 5 \times 10^{-5}$ with fluences as low as $10^{18}$ to $10^{19} \mathrm{p} \mathrm{cm}^{-2}$. In this case, ${ }^{53} \mathrm{Mn}$ would be a very latestage production in the solar system. We estimate the other nuclear effects at this level of dosage would not be large.

\subsection{On the AGB Contribution to ${ }^{129} \mathrm{I}$}

The unstable isotope ${ }^{129} \mathrm{I}$ was discovered in early solar system material by Reynolds (1960) and Jeffrey \& Reynolds (1961), and is known to be widespread in meteoritic material (cf. Swindle \& Podosek 1988). ${ }^{129} \mathrm{I}$ is marginally produced in $s$-process conditions either through neutron captures on unstable ${ }^{128} \mathrm{I}$, or through the decay of ${ }^{129} \mathrm{Te}$, produced by a minor channel of neutron addition on ${ }^{128} \mathrm{Te}$ (Fig. 8). This last nucleus is derived in turn both by neutron capture on ${ }^{127} \mathrm{Te}$ and by the electron capture decay of ${ }^{128} \mathrm{I}$. In astrophysical conditions, however, this channel is even less favored than in laboratory conditions. As shown in Tables 2-5, the net production of ${ }^{129} \mathrm{I}$ through the interplay of these two channels is always very low in AGB stars. The enhancement factors for ${ }^{127} \mathrm{I}$ are not high and the ratio ${ }^{129} \mathrm{I} /{ }^{127} \mathrm{I}$ in the $\mathrm{He}$ zone is always very low. An early solar nebula ratio of ${ }^{129} \mathrm{I} /$

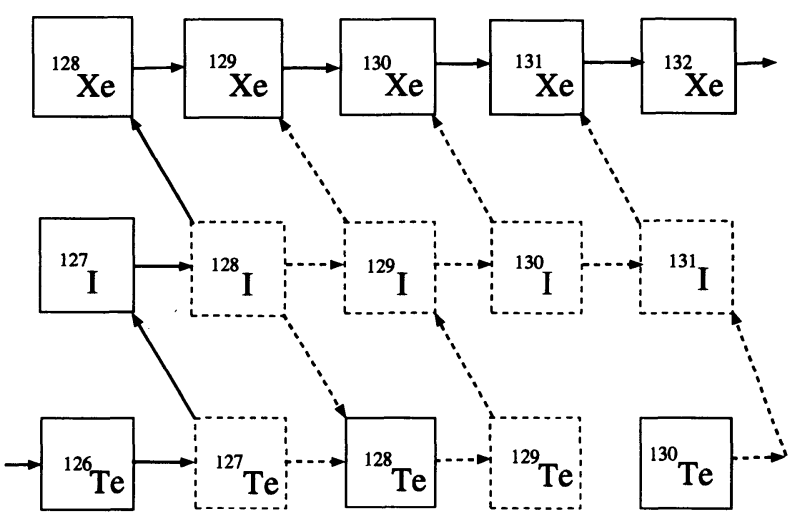

FIG. 8.-Zone of the neutron capture path involving ${ }^{129} I$

${ }^{127} \mathrm{I}=10^{-4}$ would require $M_{\mathrm{He}} / M_{\mathrm{SC}}=\sim 1, \quad 5.0 \times 10^{-2}$, $7.7 \times 10^{-3}$, and $5.2 \times 10^{-4}$ for the values of $\tau_{0}$ studied. Given the importance of ${ }^{129} \mathrm{I}$ in cosmochronologic problems, we have investigated the sensitivity of our results to the nuclear parameters involved in its synthesis, mainly the lifetimes of ${ }^{128} I$ and of ${ }^{129} \mathrm{I}$ itself. In particular, this last parameter is a sensitive function of temperature, being reduced in astrophysical conditions by six orders of magnitude with respect to the terrestrial value. We find, however, no possibility of a substantial contribution to ${ }^{129}$ I by $s$-process environments. Even allowing it to decay at its laboratory rate, the production remains marginal. In a calculation done for $\tau_{0}=0.13 \mathrm{mbarn}^{-1}$, assuming that the mean lifetime of ${ }^{129} \mathrm{I}$ in the He-zone is equal to the laboratory lifetime, we only obtain $N_{\mathrm{He}}^{129} / N_{\mathrm{He}}^{127}=8.64 \times 10^{-2}$ (in contrast to a value of $2.9 \times 10^{-3}$ when the dependence of $\bar{\tau}_{R}$ on temperature is considered). The value of $q_{\mathrm{He}}^{127} / q_{\mathrm{SC}}^{127}$ is not changed (4.49). Even in this case, the match of the early solar ratio $\left(\alpha_{129,127}=10^{-4}\right)$ would require $M_{\mathrm{He}} / M_{\mathrm{SC}}=2 \times 10^{-4}$, which is exceedingly high compared to the value for ${ }^{107} \mathrm{Pd}$ $\left(1.7 \times 10^{-6}\right.$ for this case). Moreover, this test is based on an extreme hypothesis for the decay rate, which is inconsistent with the level scheme of ${ }^{129}$ I (Lederer \& Shirley 1978) and with similar evaluations by Takahashi \& Yokoi (1987) and Cosner $\&$ Truran (1981). During the $s$-process, ${ }^{129} \mathrm{I}$ production is always inhibited by the very short lifetime of ${ }^{128} \mathrm{I}$. It follows that ${ }^{129}$ I injection from an AGB star is not a possible mechanism to explain the presence of this nuclide at the abundances found in meteorites. This result is in agreement with the conclusion by Cameron et al. (1993), who showed that ${ }^{129} \mathrm{I}$ cannot be effectively produced by an $s$-process but can be produced by the continuous addition of $r$-processed matter from supernovae during the Galactic history, in the required ratio to ${ }^{244} \mathrm{Pu}$.

\subsection{Models with Different Neutron Densities}

Because of the importance of the average neutron density, $n_{n}$, in controlling the $s$-flow in branching chains, we have investigated, in some detail, the effects of its possible variation on the production of the radioactive nuclei discussed here, in particular those presented in $\S 4.2$, whose abundance should grow with $n_{n}$.

For a given mass and composition of the thermal pulse, the neutron density increases linearly with the rate of ingestion of ${ }^{13} \mathrm{C}$. With reference to Figure 2 , this means that $n_{n}$ is controlled by the rate of expansion of the convective profile in region 2 . For a star of solar metallicity, this remains true up to a limit of a few times $10^{9} n \mathrm{~cm}^{-3}$, corresponding to an ingestion rate of a 
few $10^{-4} M_{\odot} \mathrm{yr}^{-1}$. At this point, the ingestion is so rapid that the rate of ${ }^{13} \mathrm{C}$ burning (at the typical ambient temperature of $1.5 \times 10^{8} \mathrm{~K}$ ) begins to be important, and any further increase of the ingestion rate has essentially no effect. In the case that ${ }^{13} \mathrm{C}$ would be ingested slightly later, when the temperature begins to increase, values of $n_{n}$ as high as $10^{10} n \mathrm{~cm}^{-3}$ are not impossible. Also, a reduction of the total convective mass on which ${ }^{13} \mathrm{C}$ is diluted has the obvious consequence of increasing the neutron density. In "real" AGB models, these two parameters vary smoothly from pulse to pulse: both the total pulse mass and the rate of convective growth in the region of interest decrease with increasing pulse number. In our calculations, convective growth rates cover the range from about $2 \times 10^{-4}$ $M_{\odot} \mathrm{yr}^{-1}$ in the first pulses to about $2 \times 10^{-5} M_{\odot} \mathrm{yr}^{-1}$ in the last ones, while the pulse mass changes from $\sim 3 \times 10^{-2} M_{\odot}$ to slightly less than $1 \times 10^{-2} M_{\odot}$. In the schematic pulse model we adopted for $s$-processing (see $\S 2$ ), the values found when $M_{\mathrm{C}}=0.6 M_{\odot}$ were used, roughly corresponding to averages on the TP-AGB phase. Should the ${ }^{13} \mathrm{C}$-rich pocket also be present in the first TP-AGB stages, then the first pulses would experience rather high mean neutron densities, up to a few $\times 10^{9} n \mathrm{~cm}^{-3}$. Due to the lack of a detailed model for ${ }^{13} \mathrm{C}$ production at the hydrogen/helium interface, it is still largely premature to decide whether this can occur or not. Moreover, before assuming our data on the pulse shape and mass as typical, calculations should be repeated for several different initial masses and mass-loss rates, a task which would require considerable computational effort. For the moment, one can say qualitatively that stars with masses lower than those presented here (i.e., $\sim 1 M_{\odot}$ instead of 1.5 to $3 M_{\odot}$ ), undergoing a lower number of pulses, could in principle, experience higher neutron densities. Alternatively, a high neutron density is also expected in a low-metallicity star, since, for a given (fixed) amount of ${ }^{13} \mathrm{C}$ burned, the same number of neutrons would be distributed on a lower concentration of seeds. High values of the neutron density, between $10^{9}$ and $10^{10} n \mathrm{~cm}^{-3}$, are also expected in intermediate mass stars $\left(M>3 M_{\odot}\right)$ from a more efficient activation of the ${ }^{22} \mathrm{Ne}$ neutron source (Busso et al. 1988). While this is certainly an alternative scenario to be considered, one should keep in mind that these stars are statistically less favored as a source due to their apparent rarity (Blanco, McCarty, \& Blanco 1980).

To simulate the nucleosynthesis occurring in high-neutrondensity thermal pulses, we have carried out some further exploratory calculations in which neutron captures were followed after increasing arbitrarily the rate of ${ }^{13} \mathrm{C}$ ingestion during stage 2 of our pulse scheme (see Fig. 2), to allow the average $n_{n}$ in the pulse to increase correspondingly. The results are shown in Table 6 for $n_{n}$ values in the range for $3 \times 10^{8}$ to $3.7 \times 10^{10} n \mathrm{~cm}^{-3}$. It can be seen that the values of ${ }^{135} \mathrm{Cs} /{ }^{133} \mathrm{Cs}$ and $q_{\mathrm{He}}^{133} / q_{\mathrm{SC}}^{133}$ are essentially constant and give the same $\alpha_{135,133}$. For ${ }^{182} \mathrm{Hf}$ there is a marked increase in ${ }^{182} \mathrm{Hf} /{ }^{180} \mathrm{Hf}$ with increasing $n_{n}$. In the important case of ${ }^{60} \mathrm{Fe}$, we note that while $q_{\mathrm{He}}^{56} / q_{\mathrm{SC}}^{56}$ is essentially constant, the production of ${ }^{60} \mathrm{Fe}$ increases markedly and gives ${ }^{60} \mathrm{Fe} /{ }^{56} \mathrm{Fe}$ ranging from $1.36 \times 10^{-4}$ to $2.24 \times 10^{-3}$. This gives values of $\alpha_{60,56}$ ranging from $1.0 \times 10^{-8}$ to $1.58 \times 10^{-7}$. These calculations suggest that substantial increases in the production of ${ }^{60} \mathrm{Fe}$ and ${ }^{182} \mathrm{Hf}$ may occur in AGB stars with high $n_{n}$. It follows that a more detailed study of AGB models with consideration of the mechanisms and rates of ingestion of the ${ }^{13} \mathrm{C}$ pocket may be called for if our standard model is inadequate for ${ }^{60} \mathrm{Fe}$ production.
TABLE 6

INSTANT INJECTION AND MIXING ${ }^{a}$

\begin{tabular}{cclcc}
\hline \hline \multicolumn{1}{c}{$n_{n}^{\text {Max }}$} & Isotope Ratio & \multicolumn{1}{c}{$N_{\mathrm{He}}^{R} / N_{\mathrm{He}}^{I}$} & $q_{\mathrm{He}}^{I} / q_{\mathrm{SC}}^{I}$ & \multicolumn{1}{c}{$\alpha_{R, I}$} \\
\hline $3.0 \times 10^{8} \ldots \ldots$. & ${ }^{60} \mathrm{Fe} /{ }^{56} \mathrm{Fe}$ & $1.36 \times 10^{-4}$ & 0.856 & $1.0 \times 10^{-8}$ \\
& ${ }^{135} \mathrm{Cs} /{ }^{133} \mathrm{Cs}$ & 0.522 & 0.736 & $3.26 \times 10^{-5}$ \\
& ${ }^{182} \mathrm{Hf} /{ }^{180} \mathrm{Hf}$ & 0.016 & 2.640 & $3.68 \times 10^{-6}$ \\
$3.0 \times 10^{9} \ldots \ldots$. & ${ }^{60} \mathrm{Fe} /{ }^{56} \mathrm{Fe}$ & $4.82 \times 10^{-4}$ & 0.856 & $3.50 \times 10^{-8}$ \\
& ${ }^{135} \mathrm{Cs} /{ }^{133} \mathrm{Cs}$ & 0.684 & 0.736 & $4.28 \times 10^{-5}$ \\
& ${ }^{182} \mathrm{Hf} /{ }^{180} \mathrm{Hf}$ & 0.042 & 2.600 & $9.28 \times 10^{-6}$ \\
$3.7 \times 10^{10} \ldots \ldots$. & ${ }^{60} \mathrm{Fe} /{ }^{56} \mathrm{Fe}$ & $2.24 \times 10^{-3}$ & 0.830 & $1.58 \times 10^{-7}$ \\
& ${ }^{135} \mathrm{Cs} /{ }^{133} \mathrm{Cs}$ & 0.987 & 0.714 & $6.0 \times 10^{-5}$ \\
& $182 \mathrm{Hf} /{ }^{180} \mathrm{Hf}$ & 0.118 & 2.385 & $2.39 \times 10^{-5}$ \\
\hline
\end{tabular}

a Same models as Table 2 but with simulation for various neutron densities $\left(\tau_{0}=0.03 \mathrm{mbarn}^{-1} ; M_{\mathrm{He}} / M_{\mathrm{SC}}=8.5 \times 10^{-5}\right)$. All the cases shown produce an early solar system ratio $\alpha_{107,108} \simeq 2 \times 10^{-5}$, since ${ }^{107} \mathrm{Pd}$ does not depend on $n_{n}$.

\subsection{Contributions from the $\mathrm{H}$-shell: the Case of ${ }^{26} \mathrm{Al}$}

So far we have considered the prompt enrichment of the solar nebula in nuclei produced by the He-shell. We now want to extend the analysis to ${ }^{26} \mathrm{Al}$. The presence of ${ }^{26} \mathrm{Al}$ in the early solar system (Lee, Papanastassiou, \& Wasserburg 1977) has been the basis for estimating the timescale between injection of freshly synthesized nuclei and formation and collapse of the solar nebula (cf. Wasserburg 1985). The existence of abundant ${ }^{26} \mathrm{Al}$ in the galaxy found by Mahoney et al. (1984), and confirmed by Share et al. (1985), Ballmoos, Diehl, \& Schönfelder (1987), and Diehl et al. (1993) (see also discussion by Clayton \& Leising 1987), further shows that this nuclide is widespread and is of broad astrophysical interest. The conditions for its production in AGB stars have already been discussed in $\S 2$. With the choice there of $M_{\mathrm{H}}=5 \times 10^{-4} \times N_{\mathrm{DU}}\left(N_{\mathrm{DU}}\right.$ being the number of dredge-up episodes), we can discard, in a first approximation, the residual contribution to ${ }^{26} \mathrm{Al}$ coming from the He-shell after dilution and burning, and consider only the part coming from the reservoir $M_{\mathbf{H}}$.

With the above picture in mind, we first pursue a phenomenological model for ${ }^{26} \mathrm{Al}$ addition from an AGB star, where we calculate the mass of its reservoir (assumed not to be further processed) that must be ejected to provide the required amount of this radioactive nucleus. This yields the dilution factor for this part of the $\mathrm{H}$-shell matter $\left(M_{\mathrm{H}} / M_{\mathrm{SC}}\right)$ which can then be related to the expected dilution of He-shell material once an estimate of the ratio $M_{\mathrm{H}} / M_{\mathrm{He}}$ produced by the AGB source is available. With the adopted mass loss rate (see $\S 2$ ), we have verified that stars in the mass interval 1.5 to $3 M_{\odot}$ can experience from 15 to 40 mixing episodes with dredge-up expected. Hence, assuming an average mass for the envelope of $0.5 M_{\odot}$ during $\mathrm{AGB}$, a fractional mass $M_{\mathrm{H}} / M_{\mathrm{E}} \simeq(1.5-4) \times 10^{-2}$ of $\mathrm{H}$-burning reservoir is eventually ejected by stellar winds. This has to be compared with the typical value (at $\mathrm{C} / \mathrm{O} \simeq 1$ ) of $M_{\mathrm{He}} / M_{\mathrm{E}} \simeq 2 \times 10^{-2}$. We can therefore assume, as a representative range, that $M_{\mathrm{H}} / M_{\mathrm{He}} \simeq(0.7-2)$. If the fraction of ${ }^{25} \mathrm{Mg}$ burnt by proton captures to ${ }^{26} \mathrm{Al}$ is $p$, we also have

$$
N_{\mathrm{H}}^{26}=p N_{\mathrm{H}}^{25}=p q_{\mathrm{H}}^{25} M_{\mathrm{H}}
$$

and

$N_{\mathrm{H}}^{26} / N_{\mathrm{SC}}^{27}=p q_{\mathrm{H}}^{25} M_{\mathrm{H}} /\left(q_{\mathrm{SC}}^{27} M_{\mathrm{SC}}\right)=p q_{\mathrm{H}}^{25} M_{\mathrm{H}} /\left[\left(N_{\mathrm{SC}}^{27} / N_{\mathrm{SC}}^{25}\right) q_{\mathrm{SC}}^{25} M_{\mathrm{SC}}\right]$. 
Assuming $q_{\mathrm{SC}}^{25}=q_{\mathrm{H}}^{25}$, then

$$
\alpha_{26,27}=1.26 p\left(M_{\mathrm{H}} / M_{\mathrm{SC}}\right) \text {. }
$$

For the canonical value of $\alpha_{26,27}=5 \times 10^{-5}$, and taking $p=0.8$, one gets $M_{\mathrm{H}} / M_{\mathrm{SC}}=5 \times 10^{-5}$. Using the range of $M_{\mathrm{H}} / M_{\mathrm{He}}$ derived above, one deduces: $M_{\mathrm{He}} / M_{\mathrm{SC}} \simeq(2.5-7)$ $\times 10^{-5}$, which, taking into account the uncertainties, compares well with the estimates from ${ }^{107} \mathrm{Pd}$ and ${ }^{60} \mathrm{Fe}$, provided the neutron exposure is low $\left(\tau_{0}=0.03\right.$ or $0.07 \mathrm{mbarn}^{-1}$, see Tables 2 and 3). We note that equation (11a) implies

$$
\frac{M_{\mathrm{SC}}}{M_{\mathrm{HE}}}=\frac{1}{\alpha_{26,27}}\left(\frac{M_{\mathrm{H}}}{M_{\mathrm{He}}}\right) .
$$

Using equations (3) and (11a) and neglecting the small terms, this gives

$$
\left(\frac{M_{\mathrm{H}}}{M_{\mathrm{He}}}\right) \cong \frac{\alpha_{26,27}}{\alpha_{R, I}}\left(\frac{N_{\mathrm{He}}^{R}}{N_{\mathrm{He}}^{I}}\right) \frac{q_{\mathrm{He}}^{I}}{q_{\mathrm{SC}}^{I}} .
$$

For the case of ${ }^{107} \mathrm{Pd}$ and ${ }^{108} \mathrm{Pd}$ as the reference pair and noting that the value of $N_{\mathrm{He}}^{107} / N_{\mathrm{He}}^{108} \sim \frac{1}{6}$ for all AGB models, we obtain

$$
\left(\frac{M_{\mathrm{H}}}{M_{\mathrm{He}}}\right) \cong \frac{\alpha_{26,27}}{6 \alpha_{107,108}} \frac{q_{\mathrm{He}}^{108}}{q_{\mathrm{SC}}^{108}} .
$$

The value of $\alpha_{26,27} / \alpha_{107,108}$ is about 6 if we assume an interval $\Delta_{1} \sim 10^{6} \mathrm{yr}$ for the formation of early solar nebular condensates. It follows that $M_{\mathrm{H}} / M_{\mathrm{He}} \approx q_{\mathrm{He}}^{108} / q_{\mathrm{SC}}^{108}$. This requires that for an AGB model, the ratio of $\mathrm{H}$ shell to that of $\mathrm{He}$ shell scales with $q_{\mathrm{He}}^{108} / q_{\mathrm{SC}}^{108}$. Insofar as our estimates of $M_{\mathrm{H}} / M_{\mathrm{He}} \sim 1$ are correct, it follows that only models with $q_{\mathrm{He}}^{108} / q_{\mathrm{SC}}^{108} \sim 1$ are acceptable in order to produce both ${ }^{26} \mathrm{Al}$ and ${ }^{107} \mathrm{Pd}$. This requires low $\tau_{0}$. If we had chosen an interval $\Delta_{1}$, significantly greater than $1 \mathrm{myr}$, this would demand a higher value of $M_{\mathrm{H}} / M_{\mathrm{He}}$. The choice of a low mean neutron exposure for an AGB star of solar metallicity polluting the proto-solar nebula is not in contradiction with the much higher best-fit value of $\tau_{0}=0.28$ mbarn $^{-1}$ for reproducing the main $s$-component. Indeed, as clarified by Clayton (1988), the ${ }^{13} \mathrm{C}(\alpha, n){ }^{16} \mathrm{O}$ source may potentially provide higher $\tau_{0}$ for AGB stars of lower initial metallicity than the solar one. It is then plausible to assume that the composition of the $s$-nuclei in the interstellar medium at the epoch of the Sun's formation was mainly derived from a much earlier generation of long-lived, low-mass AGB stars of initial metallicity of the order of $\frac{1}{3} Z_{\odot}$ (Gallino et al. 1988), while the single AGB star we are dealing with is more likely a short-lived star of about $3 M_{\odot}$ and the ambient local medium with about the solar metallicity.

\subsection{Contribution from Continuous Injection of AGB Matter}

Turning to the continuous production and dilution case, we may calculate $f_{I}$ (the fraction of isotope $I$ produced in AGB sources) assuming $t \simeq 10^{8} \mathrm{yr}$ for a typical cloud timescale. We explicitly discuss here the case of ${ }^{107} \mathrm{Pd}$; the other cases can be treated similarly. The nucleosynthetic parameters are given in Tables 2-5 for the different $\tau_{0}$ values. The results for all values of $\tau_{0}$ are summarized in Table 7 . For the observed ${ }^{107} \mathrm{Pd} /$ ${ }^{108} \mathrm{Pd}$ ratio $\left(2 \times 10^{-5}\right)$ and for $\tau_{0}=0.28 \mathrm{mbarn}^{-1}$, one obtains a time scale for $\dot{M}_{\mathrm{He}} / M_{\mathrm{MC}}=1.5 \times 10^{-8} \mathrm{myr}^{-1}$, and $f_{108}=1.3$ $\times 10^{-4}$. The values of $\alpha_{R, I}$ for the other nuclei are given using the value of $\dot{M}_{\mathrm{He}} / M_{\mathrm{MC}}$ determined by Pd. It can be seen that both ${ }^{26} \mathrm{Al}$ and ${ }^{60} \mathrm{Fe}$ are somewhat low in abundance. For ${ }^{26} \mathrm{Al}$ and ${ }^{60} \mathrm{Fe}$ we note that the case for $\tau_{0}=0.03 \mathrm{mbarn}^{-1}$ is the only one that yields anything close to reasonable values. These results show that while there is a substantial level of short-lived nuclei in an ISM that is continuously fed with AGB ejecta over a $10^{8} \mathrm{yr}$ timescale, these amounts are distinctly low for the short-lived ${ }^{26} \mathrm{Al}$ and ${ }^{60} \mathrm{Fe}$. Any free decay in the ISM will exacerbate this problem. As the ${ }^{60} \mathrm{Fe}$ is observed in planetary differentiates there must be some time interval in which it decays. Any reasonable estimate for this time would make it far less than the observed value.

Notice that, to produce all of the $s$-process ${ }^{108} \mathrm{Pd}(66 \%$ of $\left.{ }^{108} \mathrm{Pd}_{\odot}\right)$, i.e., $f_{108}=0.66$, one has to consider a timescale equal to the Galactic disk lifetime at the Sun's formation $\left(t_{\mathrm{G}} \simeq\right.$ $5 \times 10^{9} \mathrm{yr}$ ). Since the main $s$-process component is best fitted by $\tau_{0}=0.28$ mbarn $^{-1}$, only the last column (4) in Table 7 is applicable. The corresponding time scale can be written from equation (7): $t_{\mathrm{G}}=\left(M_{\mathrm{MC}} / \dot{M}_{\mathrm{He}}\right) q_{\mathrm{MC}}^{I} / q_{\mathrm{He}}^{I}$. For $q_{\mathrm{He}}^{I} / q_{\mathrm{MC}}^{I} \simeq 10^{3}(\mathrm{see}$ Table 5) and $t_{\mathrm{G}}=5 \times 10^{9} \mathrm{yr}$, we need $\dot{M}_{\mathrm{He}} / M_{\mathrm{MC}}=2.1 \times 10^{-7}$ $\mathrm{myr}^{-1}$. There is a substantial difference (a factor of 10$)$ between this value for the average production over Galactic history from that required to explain the production of ${ }^{107} \mathrm{Pd}$ in the proto-solar cloud with the same model $\left(\tau_{0}=0.28 \mathrm{mbarn}^{-1}\right)$. This implies that, if the continuous ejection of AGB matter is argued to explain both isotopes, ${ }^{107} \mathrm{Pd}$ and ${ }^{108} \mathrm{Pd}$, then the late addition is characterized by a rate of AGB injection (at $\tau_{0}=$ 0.28 mbarn $^{-1}$ ) much lower than the average. If one requires the late addition to be at a comparable rate of $\dot{M}_{\mathrm{He}} / M_{\mathrm{MC}}$ for the average $s$-process production over the age of the Galaxy, then this indicates a much lower value of $\tau_{0}$ for the proto-solar

\begin{tabular}{|c|c|c|c|c|}
\hline Isotope Ratio & $\begin{array}{c}N_{\mathrm{SC}}^{R} / N_{\mathrm{SC}}^{I}(\Delta=0) \\
\left(\tau_{\mathrm{o}}=0.03 \mathrm{mbarn}^{-1} ;\right. \\
\left.\dot{M}_{\mathrm{He}} / M_{\mathrm{MC}}=9.0 \times 10^{-6} \mathrm{myr}^{-1}\right)\end{array}$ & $\begin{array}{c}N_{\mathrm{sC}}^{R} / N_{\mathrm{SC}}^{I}(\Delta=0) \\
\left(\tau_{0}=0.07 \mathrm{mbarn}^{-1} ;\right. \\
\left.\dot{M}_{\mathrm{He}} / M_{\mathrm{MC}}=2.5 \times 10^{-6} \mathrm{myr}^{-1}\right)\end{array}$ & $\begin{array}{c}N_{\mathrm{SC}}^{R} / N_{\mathrm{SC}}^{I}(\Delta=0) \\
\left(\tau_{0}=0.13 \mathrm{mbarn}^{-1} ;\right. \\
\left.\dot{M}_{\mathrm{He}} / M_{\mathrm{MC}}=1.8 \times 10^{-7} \mathrm{myr}^{-1}\right)\end{array}$ & $\begin{array}{c}N_{\mathrm{SC}}^{R} / N_{\mathrm{SC}}^{I}(\Delta=0) \\
\left(\tau_{0}=0.28 \mathrm{mbarn}^{-1} ;\right. \\
\left.\dot{M}_{\mathrm{He}} / M_{\mathrm{MC}}=1.5 \times 10^{-8} \mathrm{myr}^{-1}\right)\end{array}$ \\
\hline${ }^{107} \mathrm{Pd} /{ }^{108} \mathrm{Pd} \ldots \ldots \ldots \ldots$ & $2.0 \times 10^{-5}$ & $2.0 \times 10^{-5}$ & $2.0 \times 10^{-5}$ & $2.0 \times 10^{-5}$ \\
\hline $\begin{array}{l}{ }^{205} \mathrm{~Pb} /{ }^{204} \mathrm{~Pb} \ldots \ldots \ldots \ldots \\
{ }^{93} \mathrm{Zr} /{ }^{92} \mathrm{Zr} \ldots \ldots \ldots \ldots \ldots \\
{ }^{99} \mathrm{Zc} /{ }^{100} \mathrm{Ru} \ldots \ldots \ldots \ldots \\
{ }^{60} \mathrm{Fe} /{ }^{56} \mathrm{Fe} \ldots \ldots \ldots \ldots \ldots \\
{ }^{135} \mathrm{Cs} /{ }^{133} \mathrm{Cs} \ldots \ldots \ldots \ldots \\
{ }^{182} \mathrm{Hf} /{ }^{1{ }^{180}} \mathrm{Hf} \ldots \ldots \ldots \ldots\end{array}$ & $\begin{array}{l}9.4 \times 10^{-4} \\
5.9 \times 10^{-6} \\
7.5 \times 10^{-7} \\
1.7 \times 10^{-9} \\
1.3 \times 10^{-5} \\
3.6 \times 10^{-6}\end{array}$ & $\begin{array}{l}3.6 \times 10^{-4} \\
2.0 \times 10^{-5} \\
1.3 \times 10^{-6} \\
8.2 \times 10^{-10} \\
2.9 \times 10^{-6} \\
5.6 \times 10^{-7}\end{array}$ & $\begin{array}{l}9.1 \times 10^{-5} \\
1.8 \times 10^{-5} \\
1.3 \times 10^{-6} \\
1.7 \times 10^{-10} \\
3.8 \times 10^{-6} \\
4.0 \times 10^{-7}\end{array}$ & $\begin{array}{l}6.5 \times 10^{-4} \\
1.3 \times 10^{-5} \\
1.2 \times 10^{-6} \\
4.4 \times 10^{-11} \\
1.0 \times 10^{-5} \\
4.8 \times 10^{-6}\end{array}$ \\
\hline${ }^{129} I /{ }^{127} I \ldots \ldots \ldots \ldots \ldots$ & $4.4 \times 10^{-7}$ & $1.1 \times 10^{-7}$ & $5.4 \times 10^{-8}$ & $7.1 \times 10^{-8}$ \\
\hline${ }^{26} \mathrm{Al} /{ }^{27} \mathrm{Al}^{\mathrm{a}} \ldots \ldots \ldots \ldots$ & $2.0 \times 10^{-5}$ & $5.5 \times 10^{-6}$ & $4.0 \times 10^{-7}$ & $3.3 \times 10^{-8}$ \\
\hline
\end{tabular}
addition with a corresponding decrease in $q_{\mathrm{He}}^{108}$.

TABLE 7

Live Abundances Predicted by the Continuous Ejection and Mixing over $t=10^{8}$ Years

${ }^{a}$ Assuming $\dot{M}_{\mathrm{H}} / \dot{M}_{\mathrm{He}}=2$ and $p=0.8$ (see text). 
This result was required also by the single late addition case, and is what one expects in a scenario for the Galactic enrichment of $s$-elements, in which the ${ }^{13} \mathrm{C}$ neutron source is assumed to release the same amount of neutrons independent of the stellar metallicity. Indeed, as described by Käppeler et al. (1990), assuming a typical (constant) amount of ${ }^{13} \mathrm{C}$ ingested of $4 \times 10^{-6} M_{\odot}$ in each thermal pulse of an AGB, the main component of the $s$-process is obtained in relatively lowmetallicity stars $\left(Z=Z_{\odot} / 3\right)$, while for higher metallicities the mean neutron exposure roughly scales as $1 / Z$. Hence, a selfconsistent model for radioactive ${ }^{107} \mathrm{Pd}$ and stable ${ }^{108} \mathrm{Pd}$ by continuous injection over a long time may be possible.

For the case of continuous production of ${ }^{26} \mathrm{Al}$ and injection into a cloud, the equations corresponding to (10) and (11) are

$$
\dot{N}_{\mathrm{H}}^{26}=p \frac{N_{\mathrm{H}}^{25}}{M_{\mathrm{H}}} \dot{M}_{\mathrm{H}}-\frac{N_{\mathrm{SC}}^{26}}{\bar{\tau}_{26}} \approx 0
$$

or

$$
N_{\mathrm{SC}}^{26}=\bar{\tau}_{26} p q_{\mathrm{H}}^{25} \dot{M}_{\mathrm{H}}
$$

and

$$
N_{\mathrm{SC}}^{26} / N_{\mathrm{SC}}^{27}=\bar{\tau}_{26} p q_{\mathrm{H}}^{25}\left(\dot{M}_{\mathrm{H}} / q_{\mathrm{SC}}^{27} M_{\mathrm{SC}}\right)
$$

Again assuming solar abundances for ${ }^{25} \mathrm{Mg}$ in the H-reservoir and ${ }^{27} \mathrm{Al}$ in the SC-reservoir we have

$$
N_{\mathrm{MC}}^{26} / N_{\mathrm{MC}}^{27}=1.1\left(\dot{M}_{\mathrm{H}} / M_{\mathrm{SC}}\right) \text {. }
$$

Taking $\dot{M}_{\mathrm{H}} / M_{\mathrm{He}}=2$ and the corresponding value of $\dot{M}_{\mathrm{He}} / M_{\mathrm{SC}}$, we obtain the values in the last row of Table 7 for the different values of $\tau_{0}$. It can be seen that the only values of ${ }^{26} \mathrm{Al} /{ }^{27} \mathrm{Al}$ that approach the observed value of $5 \times 10^{-5}$ in the early solar nebular materials is for $\tau_{0}=0.03$ mbarn $^{-1}$. However, this ratio does not provide for any time interval between the isolation of the proto-solar nebula and condensation.

It is of interest to consider the general mechanism of ${ }^{26} \mathrm{Al}$ formation in AGB stars for the source of the $\sim 4 M_{\odot}$ of ${ }^{26} \mathrm{Al}$ present in the Galaxy corresponding to a steady state injection of $3.6 \times 10^{-6} M_{\odot}$ of ${ }^{26} \mathrm{Al}$ per year into the ISM. This matter has been discussed in a more sophisticated argument by Forestini et al. (1991). From recent observations, the Galactic ${ }^{26} \mathrm{Al}$ source does not appear to be located in the close neighborhood of the Galactic center as surmised by Ballmoos et al. (1989) but rather is broadly distributed (Diehl et al. 1993). From equation (12) the mass rate of injection of ${ }^{26} \mathrm{Al}$ is $25 p q_{\mathrm{H}}^{25} \dot{M}_{\mathrm{H}}$ where $\dot{M}_{\mathrm{H}}$ is the rate of injection of material from the $\mathrm{H}$ burning zone where ${ }^{25} \mathrm{Mg}$ is converted to ${ }^{26} \mathrm{Al}$. We obtain $25 p q_{\mathrm{H}}^{25} \dot{M}_{\mathrm{H}}=8$ $\times 10^{-5} \dot{M}_{\mathrm{H}}$ solar masses of ${ }^{26} \mathrm{Al}$ per unit time. If we equate this with the observationally inferred injection rate of ${ }^{26} \mathrm{Al}$ of $3.6 \times 10^{-6} M_{\odot}$ of ${ }^{26} \mathrm{Al}$ per year, this corresponds to the ejection of $\dot{M}_{\mathrm{H}} \sim 4 \times 10^{-2} M_{\odot} \mathrm{yr}^{-1}$ of $\mathrm{H}$ zone burnt material for the Galaxy. Insofar as the $\mathrm{H}$ zone constitutes $\sim 1 \%$ to $4 \%$ of the total envelope of a typical AGB, this would require the net ejection of $A G B$ matter of 1-4 $M_{\odot}$ per year in the galaxy from stars passing through the thermally pulsing AGB phase. Only in this case could AGB stars provide the source of ${ }^{26} \mathrm{Al}$ in the galaxy at the present time. The current estimate for the return of matter to the ISM in the galaxy is $\sim 0.4 M_{\odot}$ yr. While AGB stars appear to require a somewhat higher rate of return of matter than current estimates, in order for them to be the main source for Galactic ${ }^{26} \mathrm{Al}$, we are not clear as to the reliability of the current estimated value for the return rate. This conclusion is in disagreement with the evaluation by Forestini et al. 1991, who conclude that AGB sources are far too feeble a source for
Galactic ${ }^{26} \mathrm{Al}$. Other sources of the Galactic ${ }^{26} \mathrm{Al}$ have been proposed such as Wolf-Rayet stars and novae. A recent discussion of the possible sources is summarized by Clayton $\&$ Leising (1987) and Prantzos (1991).

As for ${ }^{60} \mathrm{Fe}$ in the galaxy from an AGB-like source, we have from equations (8) and (12)

$$
N_{\mathrm{MC}}^{60}=\left(\frac{N_{\mathrm{He}}^{60}}{M_{\mathrm{He}}}\right) \dot{M}_{\mathrm{He}} \bar{\tau}_{60}
$$

or

$$
\frac{N_{\mathrm{MC}}^{60}}{N_{\mathrm{MC}}^{26}}=\left(\frac{N_{\mathrm{He}}^{60}}{M_{\mathrm{He}}}\right) \frac{\dot{M}_{\mathrm{He}} \bar{\tau}_{60}}{\dot{M}_{\mathrm{H}} p q_{\mathrm{H}}^{25} \bar{\tau}_{26}}=\left(\frac{N_{\mathrm{He}}^{60}}{N_{\mathrm{He}}^{56}}\right) \frac{q_{\mathrm{He}}^{56} \dot{M}_{\mathrm{He}} \bar{\tau}_{60}}{q_{\mathrm{H}}^{25} \dot{M}_{\mathrm{H}} p \bar{\tau}_{26}} .
$$

For $\tau_{0}=0.03$ mbarn $^{-1}$, this gives $N_{\mathrm{MC}}^{60} / N_{\mathrm{MC}}^{26} \cong 10^{-3}$. It follows that an AGB source for ${ }^{26} \mathrm{Al}$ in the galaxy will only be a negligible source of ${ }^{60} \mathrm{Fe}$ and the associated $\gamma$ lines from ${ }^{60} \mathrm{Co}$. This is in accord with the observations of Mahoney et al. (1984).

\section{EPOCHS OF FORMATION-EARLY AND LATER}

In our previous discussion we have dominantly considered the initial abundances of radioactive nuclei produced by AGB sources in the molecular cloud prior to solar system formation using ${ }^{107} \mathrm{Pd}$ as a guide. As noted, the abundances of these nuclei at later times will be changed due to radioactive decay. We now consider the different epochs in which the early nebular and planetary materials formed that contained these nuclei. Our choice of ${ }^{107} \mathrm{Pd}$ was based in part on the fact that its mean life of $9.4 \times 10^{6} \mathrm{yr}$ is sufficiently long so that the initial abundance would not be drastically altered over a time interval of several millions of years. We now consider two time epochs following the initial state. We take $\Delta_{1}$ as the time interval between the assumed initial state of the ISM and the isolation of the proto-solar material, its collapse to form the solar system and the formation of early solar nebular condensates. The isotope ${ }^{26} \mathrm{Al}$ has been found almost exclusively in material that is considered to be early nebular condensates and indicates a time interval $\Delta_{1}$ of at most a few million years (Wasserburg 1985). Subsequent to this, there is a time interval, $\Delta_{2}$, at which small planetary bodies are aggregated, melted, and differentiated. The isotopes ${ }^{107} \mathrm{Pd}$ and ${ }^{60} \mathrm{Fe}$ are found in such early planetary materials that must represent objects several kilometers in size. The formation of terrestrial planets occurs in a much later epoch of 50-100 myr (Wasserburg 1985). These timescales are in part estimated by Safronov $(1969$, p. 206) through calculations of the collisional and accretional history in the solar accretion disk.

From consideration of the models presented above, it appears that only cases with low $\tau_{0}$ will have appropriate yields of ${ }^{26} \mathrm{Al}$ and ${ }^{60} \mathrm{Fe}$ compared with ${ }^{107} \mathrm{Pd}$. Taking the initial state for the instant contamination model to be determined by $\tau_{0}=0.03$ mbarn $^{-1}$ with the associated values of $N_{\mathrm{He}}^{R} / N_{\mathrm{He}}^{I}, q_{\mathrm{He}}^{I} / q_{\mathrm{SC}}^{I}$ and then assigning values of $\Delta_{1}$ and $\Delta_{2}$, we calculate the original value for $\operatorname{Pd}_{0}$ to be $\alpha_{107,108}=2 \times 10^{-5}$ $\exp \left[\left(\Delta_{1}+\Delta_{2}\right) / \bar{\tau}_{107}\right]$. This then determines a new value of $M_{\mathrm{He}} / M_{\mathrm{SC}}$ (somewhat greater than given in Table 2). The values of all other $\alpha_{R, I}$ are then determined. If we fix $M_{\mathrm{H}} / M_{\mathrm{He}}$ we then obtain $\alpha_{26,27}$. Table 8 exhibits the cases: (a) $\Delta_{1}=1 \mathrm{myr}, \Delta_{2}=$ $4 \mathrm{myr}$; and (b) $\Delta_{1}=1 \mathrm{myr}, \Delta_{2}=9 \mathrm{myr}$. Here we have taken $M_{\mathrm{H}} / M_{\mathrm{He}}=2$. The value of ${ }^{107} \mathrm{Pd} /{ }^{108} \mathrm{Pd}$ at $\Delta_{1}+\Delta_{2}$ is again taken to be $2 \times 10^{-5}$ and is generally consistent with the many observations of iron meteorites (see Kelly \& Wasserburg 1978; 
TABLE 8

Relative Abundances of Radioactive Nuclei at Different Epochs

\begin{tabular}{|c|c|c|c|c|}
\hline \multirow[b]{3}{*}{ ISOTOPE RATIO } & \multicolumn{4}{|c|}{$\alpha_{R, I}\left(\Delta_{1} \Delta_{2}\right)$} \\
\hline & \multicolumn{2}{|c|}{$\begin{array}{c}\tau_{0}=0.03 \text { mbarn }^{-1} ; \Delta_{2}=4 \mathrm{myr} \\
\Delta_{1}=1 \mathrm{myr} \\
M_{\mathrm{He}} / M_{\mathrm{SC}}=1.46 \times 10^{-4}\end{array}$} & \multicolumn{2}{|c|}{$\begin{array}{c}\tau_{0}=0.03 \mathrm{mbarn}^{-1} ; \Delta_{2}=9 \mathrm{myr} ; \\
\Delta_{1}=1 \mathrm{myr} \\
M_{\mathrm{He}} / M_{\mathrm{SC}}=2.5 \times 10^{-4}\end{array}$} \\
\hline & $1 \mathrm{myr}$ & $5 \mathrm{myr}$ & $1 \mathrm{myr}$ & $10 \mathrm{myr}$ \\
\hline${ }^{107} \mathrm{Pd} /{ }^{108} \mathrm{Pd} \ldots \ldots \ldots \ldots$ & $3.1 \times 10^{-5}$ & $2 \times 10^{-5}$ & $5.2 \times 10^{-5}$ & $2 \times 10^{-5}$ \\
\hline $\begin{array}{l}{ }^{205} \mathrm{~Pb} /{ }^{204} \mathrm{~Pb} \ldots \ldots \ldots \ldots \\
{ }^{93} \mathrm{Zr} /{ }^{92} \mathrm{Zr} \ldots \ldots \ldots \ldots \ldots \\
{ }^{99} \mathrm{Tc} /{ }^{100} \mathrm{Ru} \ldots \ldots \ldots \ldots \\
{ }^{60} \mathrm{Fe} /{ }^{56} \mathrm{Fe} \ldots \ldots \ldots \ldots \ldots \\
{ }^{135} \mathrm{Cs} /{ }^{133} \mathrm{Cs} \ldots \ldots \ldots \ldots \\
{ }^{182} \mathrm{Hf} /{ }^{180} \mathrm{Hf} \ldots \ldots \ldots \ldots \\
{ }^{129} \mathrm{I} /{ }^{127} \mathrm{I} \ldots \ldots \ldots \ldots \ldots\end{array}$ & $\begin{array}{l}6.5 \times 10^{-4} \\
6.2 \times 10^{-6} \\
1.6 \times 10^{-6} \\
7.8 \times 10^{-9} \\
3.8 \times 10^{-5} \\
4.1 \times 10^{-6} \\
2.9 \times 10^{-7}\end{array}$ & $\begin{array}{l}5.4 \times 10^{-4} \\
4.5 \times 10^{-6} \\
3.9 \times 10^{-12} \\
1.3 \times 10^{-9} \\
1.5 \times 10^{-5} \\
3.0 \times 10^{-6} \\
2.5 \times 10^{-7}\end{array}$ & $\begin{array}{l}1.1 \times 10^{-3} \\
4.7 \times 10^{-5} \\
2.6 \times 10^{-6} \\
1.3 \times 10^{-8} \\
6.4 \times 10^{-5} \\
7.0 \times 10^{-6} \\
5.0 \times 10^{-7}\end{array}$ & $\begin{array}{l}7.4 \times 10^{-4} \\
7.8 \times 10^{-7} \\
6.5 \times 10^{-19} \\
2.2 \times 10^{-10} \\
7.9 \times 10^{-6} \\
3.5 \times 10^{-6} \\
3.0 \times 10^{-7}\end{array}$ \\
\hline$\left[{ }^{26} \mathrm{Al} /{ }^{27} \mathrm{Al} \ldots \ldots \ldots \ldots\right.$ & $\begin{array}{c}1.0 \times 10^{-4} \\
\left(M_{\mathrm{H}} / M_{\mathrm{He}} \cong 2\right)\end{array}$ & $\left.2.6 \times 10^{-6}\right]$ & $\begin{array}{c}2.0 \times 10^{-4} \\
\left(M_{\mathrm{H}} / M_{\mathrm{He}} \cong 2\right)\end{array}$ & $\left.5.6 \times 10^{-8}\right]$ \\
\hline
\end{tabular}

Kaiser \& Wasserburg 1983; Chen \& Wasserburg 1983, 1990). The ${ }^{26} \mathrm{Al} /{ }^{27} \mathrm{Al}$ abundance at $1 \mathrm{myr}$ is in very good agreement with the observations on calcium aluminum rich inclusions that are interpreted as early nebular condensates. The model is also in agreement with the very low abundance or absence of ${ }^{26} \mathrm{Al}$ observed in later objects at $\Delta_{1}+\Delta_{2}$. To obtain better agreement with the observations, it would be sufficient to assign these condensates to a time $\Delta_{1}^{\prime}=1.7 \mathrm{myr}$ or by dropping $M_{\mathrm{H}} / M_{\mathrm{He}}$ to 1 .

The high abundance of ${ }^{205} \mathrm{~Pb}$ calculated for the AGB model suggests that this should provide an important test; however, there is some complexity due to the increased rates of electron capture of this nucleus under different astrophysical conditions and our estimates are considered upper limits using the laboratory lifetimes (see Fig. 9). The existing observational database in meteorites is limited. The early work by Anders \& Stevens (1960) is not of sufficient precision $(\sim 1 \%)$ to allow any comparison. The more recent studies by Ostic et al. (1969) and by Huey \& Kohman (1972) report ${ }^{205} \mathrm{Tl} /{ }^{203} \mathrm{Tl}$ measurements at a level of $\sim 2$ per million, where the dominant uncertainty comes from instrumental mass fractionation that cannot be easily eliminated. These workers conclude that $\left({ }^{205} \mathrm{~Pb} /{ }^{204} \mathrm{~Pb}\right)_{\odot} \lesssim$ $10^{-4}$. There is also a serious problem of lead contamination that would give erroneously high ${ }^{204} \mathrm{~Pb} /{ }^{203} \mathrm{Tl}$ ratios. The only recent data of high quality in both $\mathrm{Tl}$ and $\mathrm{Pb}$ were reported by Chen \& Wasserburg (1987) and which have uncertainties in ${ }^{205} \mathrm{Tl} /{ }^{203} \mathrm{Tl}$ of $\sim 1$ per million. The samples they studied had

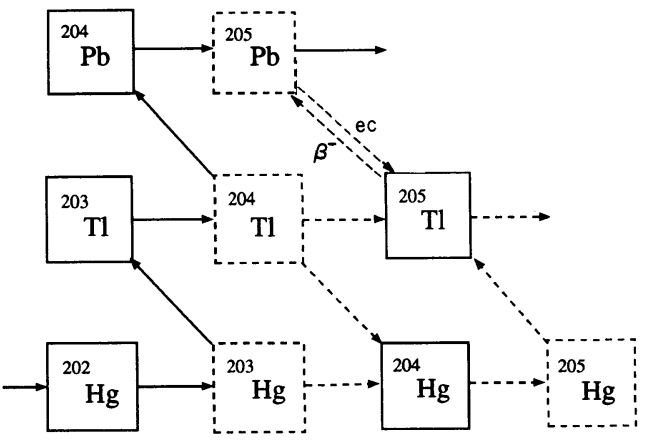

Fig. 9.-Zone of the neutron capture path involving ${ }^{205} \mathrm{~Pb}$. Note the $E C / \beta^{-1}$ transitions between ${ }^{205} \mathrm{~Pb}$ and ${ }^{205} \mathrm{Tl}$ (see Yokoi et al. 1985). primordial $\mathrm{Pb}$ so that lead contamination is not an issue. One meteorite, Nantan, shows an enrichment of 3.7 per million in ${ }^{205} \mathrm{Tl}$, which would correspond to ${ }^{205} \mathrm{~Pb} /{ }^{204} \mathrm{~Pb} \sim 3 \times 10^{-4}$. These workers consider this a hint but not a clear demonstration of ${ }^{205} \mathrm{~Pb}$ in situ decay. It is seen that, overall, the existing data indicate ${ }^{205} \mathrm{~Pb} /{ }^{204} \mathrm{~Pb}$ is low compared to our model. It is evident that a more intensive and thorough search for ${ }^{205} \mathrm{~Pb}$ is required with particular attention paid to samples which had ${ }^{107} \mathrm{Pd}$ present, as well as a better theoretical evaluation of ${ }^{205} \mathrm{~Pb}$ survivability in the interpulse period (Yokoi et al. 1985).

In contrast, while ${ }^{135} \mathrm{Cs}$ is produced in a branching chain, the calculated production appears robust and not very sensitive to neutron density. The only evidence for ${ }^{135} \mathrm{Cs}$ in the solar system is the discovery by McCulloch \& Wasserburg (1978) of a shortfall in ${ }^{135} \mathrm{Ba}$ by $10^{-4}$ that might be attributed to a holdup due to the presence of ${ }^{135} \mathrm{Cs}$. However, this is also only a hint as no correlation of isotopic shifts with the $\mathrm{Cs} / \mathrm{Ba}$ ratio have been found (see Wasserburg, Papanastassiou, \& Lee 1979).

${ }^{60} \mathrm{Fe} /{ }^{56} \mathrm{Fe}$ is lower (at $5 \mathrm{myr}$ ) by a factor of $\sim 3$ than the value reported by Shukolyukov \& Lugmair (1993) for a planetary differentiate. The difference is not very large, when the effect of cross section uncertainties on the predicted ${ }^{60} \mathrm{Fe}$ abundance is taken into account. However, if one accepts a factor of 3 as a significant discrepancy, it would suggest that the differentiate formed earlier or that the present AGB model is not adequate. As noted in our discussion of neutron density, if one increases the value $n_{n}$ to $\sim 3 \times 10^{9} n \mathrm{~cm}^{-3}$, this would increase the initial ${ }^{60} \mathrm{Fe} /{ }^{56} \mathrm{Fe}$ value by the right factor. It is evident that a more thorough study of neutron densities in these AGB models is required with due consideration of realistic stellar processes for the formation of the ${ }^{13} \mathrm{C}$ neutron source. Insofar as the representation for $\Delta_{1} \cong 1 \mathrm{myr}, \Delta_{2}=4 \mathrm{myr}$ appears to approximate consistent results for three short-lived nuclei, the other isotopic abundances in Table 8 should be a basis for predicting future observations.

If we consider $\Delta_{1}+\Delta_{2}=10 \mathrm{myr}$, then we find that the ratio ${ }^{26} \mathrm{Al} /{ }^{27} \mathrm{Al}$ is a factor of 4 higher than observed but this is readily accounted for by changing $\Delta_{1}$ to $2.5 \mathrm{myr}$ or lowering the value of $M_{\mathrm{H}} / M_{\mathrm{He}}$ (i.e., assuming a lower mass star with fewer dredge-up episodes). As for ${ }^{60} \mathrm{Fe} /{ }^{56} \mathrm{Fe}$, there is a major decrease in its value to $2.2 \times 10^{-10}$ at 10 myr. This is far below the observed value $(\times 20)$. In order to produce enough ${ }^{60} \mathrm{Fe}$, it 
would be necessary to assume a neutron density of $\sim 3 \times 10^{10}$ $n \mathrm{~cm}^{-3}$ (see Table 6). The ratio ${ }^{205} \mathrm{~Pb} /{ }^{204} \mathrm{~Pb}$ is similar to the case $\Delta_{1}+\Delta_{2}=5$ myr.

\section{CONCLUSIONS}

We have carried out calculations of the yields of nuclei produced within AGB stars in the mass range 1.5-3 $M_{\odot}$ and considered the nuclei ejected into the ISM by solar winds from these sources. We find that the short-lived nuclei ${ }^{107} \mathrm{Pd},{ }^{205} \mathrm{~Pb}$, and ${ }^{93} \mathrm{Zr}$ are efficiently produced by nonbranching $s$-process reactions in all models of AGB stars. The survivability of ${ }^{205} \mathrm{~Pb}$ during the interpulse period remains a difficult problem. The ratios of the radioactive nuclides relative to stable index isotopes $\left(N_{\mathrm{He}}^{R} / N_{\mathrm{He}}^{I}\right)$ in the $s$-chains are all approximately constant independent of the model AGB parameters. The only significant variations are in the enhancement factors, $q_{\mathrm{He}}^{I} / q_{\mathrm{SC}}^{I}$, of the isotope $I$ relative to that in the ISM. Larger neutron exposures give larger enhancement factors but the resulting ratios $N_{\mathrm{He}}^{R} / N_{\mathrm{He}}^{I}$ in the helium shell are independent of the neutron density $n_{n}$ as shown by Cameron (1993). The shortlived nuclei ${ }^{60} \mathrm{Fe},{ }^{135} \mathrm{Cs}$, and ${ }^{182} \mathrm{Hf}$ produced in branching reactions are also produced in these models but more weakly. Of these, ${ }^{135} \mathrm{Cs}$ is produced regularly and ${ }^{135} \mathrm{Cs} /{ }^{133} \mathrm{Cs}$ is not very sensitive to $n_{n}$. The nuclei ${ }^{182} \mathrm{Hf}$ and ${ }^{60} \mathrm{Fe}$ are sensitive to the neutron density in the AGB models. In particular, the production of ${ }^{60} \mathrm{Fe}$ is greatly enhanced with $n_{n}$. The neutron density is related to the rate of ingestion of ${ }^{13} \mathrm{C}$ into the He zone. Using the isotopic pair ${ }^{107} \mathrm{Pd} /{ }^{108} \mathrm{Pd}$ as a reference value for determining the amount of AGB material that must be added to the ambient ISM, the abundances of all other isotopes were calculated. The results show that in our standard AGB models, ratios $\alpha_{R, I}$ for a contaminated solar cloud for ${ }^{205} \mathrm{~Pb} /{ }^{204} \mathrm{~Pb},{ }^{93} \mathrm{Zr} /{ }^{92} \mathrm{Zr}$, and ${ }^{135} \mathrm{Cs} /{ }^{133} \mathrm{Cs}$ all lie in the range of $\sim 10^{-5}$ to $10^{-4}$. In all cases ${ }^{205} \mathrm{~Pb} /{ }^{204} \mathrm{~Pb}$ has the highest ratio. Virtually no ${ }^{129} \mathrm{I}$ is produced. ${ }^{182} \mathrm{Hf} /{ }^{180} \mathrm{Hf}$ is found to lie between $8 \times 10^{-7}$ and $4 \times 10^{-6} \cdot{ }^{60} \mathrm{Fe} /{ }^{56} \mathrm{Fe}$ is found to lie between $2 \times 10^{-10}$ and $7 \times 10^{-9}$. Parametrically increasing the rate of ${ }^{13} \mathrm{C}$ ingestion in the He zone in our AGB model, increased the ratio of ${ }^{60} \mathrm{Fe} /{ }^{56} \mathrm{Fe}$ in the He zone from $1 \times 10^{-4}$ to $2 \times 10^{-3}$ for $\tau_{0}=0.03 \mathrm{mbarn}^{-1}$. Part of the $\mathrm{H}$ burning zone produces ${ }^{26} \mathrm{Al}$ from ${ }^{25} \mathrm{Mg}$ that can be ejected into the ISM. We find that the AGB stellar winds contain about the same amount of material $\left(M_{\mathrm{H}}\right)$ from $\mathrm{H}$-processed zones where ${ }^{26} \mathrm{Al}$ is present and preserved as it does of material $\left(M_{\mathrm{He}}\right)$ from He-processed zones where $s$-process nuclei are synthesized (i.e., $\left.M_{\mathrm{H}} \sim M_{\mathrm{He}}\right)$. With increasing $\tau_{0}$, the mass of He shell required rapidly decreases due to the large values of the enhancement factor for Pd with increasing $\tau_{0}$. It is also noted that higher values of $\tau_{0}$ yield lower ${ }^{60} \mathrm{Fe} /{ }^{56} \mathrm{Fe}$. A self-consistent model that seeks to explain both ${ }^{107} \mathrm{Pd}$ and ${ }^{26} \mathrm{Al}$ requires a low $\tau_{0} \sim 0.03$ mbarn $^{-1}$. This also provides substantial ${ }^{60} \mathrm{Fe}$.

If we take into account the time differences between formation of planetary bodies, of earlier nebular condensates and the time of rapid contamination of the ISM, then the following conclusions are reached (see Table 8). For the con tamination of ambient ISM with ejecta from a single AGB star of $1.5 M_{\odot}$, a mass of $1.5 \times 10^{-4} M_{\odot}$ of He shell material with $\tau_{0} \sim 0.03$ mbarn $^{-1}$ is required to contaminate one solar mass of a protosolar cloud to produce the ${ }^{107} \mathrm{Pd} /{ }^{108} \mathrm{Pd}$ observed in planetary differentiates in the solar system at $5 \mathrm{myr}$ after contamination of the ISM. This would also provide the several other nuclei mentioned above that have not yet been observed. The ratio of ${ }^{60} \mathrm{Fe} /{ }^{56} \mathrm{Fe}$ at 1 myr after contamination of the cloud is well within the range reported by Shukolyukov \& Lugmair (1993). However, the value at 5 myr after contamination is a factor of $\sim 3$ less. The abundance of ${ }^{26} \mathrm{Al} /{ }^{27} \mathrm{Al}$ is in excellent agreement with the observations of Lee et al. (1977). If we consider that the planetary differentiates containing ${ }^{107} \mathrm{Pd}$ and ${ }^{60} \mathrm{Fe}$ were formed at $10 \mathrm{myr}$ after contamination of the ISM, it is found that ${ }^{60} \mathrm{Fe} /{ }^{56} \mathrm{Fe}$ at that time is a factor of 20 below the observed value. The ratios ${ }^{205} \mathrm{~Pb} /{ }^{204} \mathrm{~Pb}$ and ${ }^{182} \mathrm{Hf} /$ ${ }^{180} \mathrm{Hf}$ are essentially unchanged but ${ }^{93} \mathrm{Zr} /{ }^{92} \mathrm{Zr}$ decreases by a factor of 6. It can be seen that general agreement is obtained with the key observations if the timescale $\Delta_{1}+\Delta_{2}$ is less than 5 myr. If a timescale of 10 myr is required, there is a serious discrepancy with ${ }^{60} \mathrm{Fe}$. If we consider a possible increase in neutron density produced by rapid ingestion of ${ }^{13} \mathrm{C}$, then it is possible to find a self-consistent solution that will allow a timescale of $\sim 10$ myr between injection and the formation of planetary differentiates. In contrast to a single injection model, it is found that continuous ejection of AGB material into the ISM over a long timescale will not match the observations of ${ }^{26} \mathrm{Al}$ and ${ }^{60} \mathrm{Fe}$.

We conclude that prompt contamination of the medium from which the proto-solar cloud collapsed by an AGB source with $\tau_{0}=0.03$ mbarn $^{-1}$, but with elevated neutron density, would quantitatively provide all the well-documented radioactive nuclei that can be related to such sources as are observed in the early solar system. Key tests of this model will be the abundance of ${ }^{135} \mathrm{Cs},{ }^{205} \mathrm{~Pb}$ and ${ }^{182} \mathrm{Hf}$ that are clearly correlated with ${ }^{107} \mathrm{Pd}$ in a model involving s-processing. Fluctuations in the degree of homogenization of the ejecta in the ISM will cause isotopic variations with $s$-process excesses and apparent $r$-process excesses at the level of $\sim 1$ to 0.1 parts in $10^{4}$. In addition to the $s$-process nuclei, there are larger effects in ${ }^{22} \mathrm{Ne}$, which is strongly produced in the He-shell where ${ }^{14} \mathrm{~N}$ is rapidly transformed to ${ }^{22} \mathrm{Ne}$. The He-shell has $N_{\mathrm{He}}^{22} / N_{\mathrm{He}}^{20}=$ 11.5 and $q_{\mathrm{He}}^{20} / q^{\mathrm{SC} 20} \approx 1$, which are independent of $\tau_{0}$. This constitutes a $1 \%$ increase in ${ }^{22} \mathrm{Ne} /{ }^{20} \mathrm{Ne}$ in the solar cloud for our model with $\tau_{0}=0.03$ mbarn $^{-1}$, a significant but not dominant change.

A detailed investigation of neutron densities in the He zone is necessary to establish whether sufficiently high $n_{n}$ values (from $10^{9}$ to $10^{10} n \mathrm{~cm}^{-3}$ ) can be reached to produce ${ }^{60} \mathrm{Fe}$ at the levels indicated by the observations of Shukolyukov \& Lugmair (1993). The process of mixing AGB ejecta into the ISM or a planetary nebula and the ISM will also require careful scrutiny.

In the approach presented here, we have treated the problem of short-lived nuclei in the proto-solar ISM as resulting from a small amount of contamination by AGB ejecta $\left(\sim 10^{-2} M_{\odot}\right.$ of total AGB ejecta from the $\mathrm{H}$ envelope and the $\mathrm{He}$ shell to one $M_{\odot}$ of ISM). This model requires a local contribution in the neighborhood of the protosolar nebula. Another possibility is that the AGB completes its evolution and explodes forming a planetary nebula. The scale factor of dilution remains unchanged in our calculation. The total mass of ejected matter from the AGB through the planetary nebula phase is $\sim 1 M_{\odot}$. The formation of the solar nebula is, in this case, considered to occur in a region of $\sim 100 M_{\odot}$ of nearby gas and dust in the ISM, spanned by the planetary nebular ejecta. Alternatively, the dilution factor could just be a matter of the solid angle subtended by the solar cloud as seen by the polluting source. The possible role of a planetary nebula as the environment or trigger for forming the solar system and for injecting fresh nuclei has been discussed by Cameron $(1984,1985)$. The 
assumed effectiveness of mixing the short-lived nuclei may be due either to the slow cool wind typical of AGB stars or to the final fast winds associated with the planetary nebula phase. In any case, the model of AGB contributions to a molecular cloud requires some special circumstances: in particular, the probability of finding a pulsing or terminal phase AGB star near $(\sim 1 \mathrm{pc})$ or associated with the proto-solar cloud remains to be investigated.

The authors are thankful to A. G. W. Cameron for his constructive and instructive comments on this report and for his work in setting the stage and laying out the script. Icko Iben was kind enough to lead us through the maze of stellar evolution pulse by pulse. We are indebted to John Lattanzio, Alessandro Chieffi, Marco Limongi, and Oscar Straniero for letting us know the results of their evolutionary models in advance of publication. Thanks are also due to D. D. Clayton and J. Mould for many useful discussions on these and related topics. We acknowledge the use of computer facilities of the Istituto di Fisica Generale, Universita di Torino, and the Osservatorio di Torino. Valuable discussions with I.-J. Sackmann are acknowledged. T. Tombrello's Gordon Conference comments were stimulating. Comments and criticisms by G. Lugmair were much appreciated. This work was supported by NASA grant NAGW-3337. Division Contribution 5221(790).
Anders, E., \& Grevesse, N. 1989, Geochim. Cosmochim. Acta, 53, 197 Anders, E., \& Stevens, C. M. 1960, J. Geophys. Res., 65, 3043 Ballmoos, P. V., Diehl, R., \& Schönfelder, V. 1987, ApJ, 318, 659 Beer, H., Voss, F., \& Winters, F. F. 1992, ApJS, 80, 403

Birck, J. L., \& Allègre, C. J. 1985, Geophys. Res. Lett., 12, 745

Blake, J. B., Lee, T., \& Schramm, D. N. 1973, Nature Phys. Sci., 242, 98

Blake, J. B., \& Schramm, D. N. 1975, ApJ, 197, 165

Blanco, V. M., McCarty, M. F., \& Blanco, B. M. 1980, ApJ, 242, 938

Boothroyd, A. I., \& Sackmann, I.-J. 1988, ApJ, 328, 671

Busso, M., Gallino, R., Lambert, D. L., Raiteri, C. M., \& Smith, V. V. 1992, ApJ, 399, 218

. 1994, in preparation

Busso, M., Chieffi, A., Gallino, R., Limongi, M., Raiteri, C. M., \& Straniero, O. 1993, in IAU Symp. 155, Planetary Nebulae, ed. R. Weinberg \& A. Acker (Dordrecht: Kluwer), 361

Busso, M., Picchio, G., Gallino, R., \& Chieffi, A. 1988, ApJ, 326, 196

Cameron, A. G. W. 1984, Icarus, 6, 416

. 1984, in Essays in Nuclear Physics, ed. C. A. Barnes, D. D. Clayton, \&

D. N. Schramm (Cambridge: Cambridge Univ. Press), 23

1985, in Protostars and Planets II, ed. D. C. Black \& M. S. Matthews

(Tucson: Univ. Arizona Press), 1073

. 1993, in Protostars and Planets III, ed. E. H. Levy, \& J. I. Lunine (Tucson: Univ. Arizona Press), 47

Cameron, A. G. W., Tielemann, F.-K., \& Cowan, J. J. 1993, Phys. Rep., 227, 283

Caughlan, G. R., \& Fowler, W. A. 1988, Atomic Data Nucl. Data, 40, 283

Chen, J. H., \& Wasserburg, G. J. 1983, Geochim. Cosmochim. Acta, 47, 1725 1987, Lunar Planet. Sci. Conf. XVIII (Houston: Lunar and Planetary Inst., USRA), 165

1990, Geochim. Cosmochim. Acta, 54, 1729

Chieffi, A., Limongi, M., \& Straniero, O. 1994, in preparation

Clayton, D. D. 1988, MNRAS, 234,

Clayton, D. D., Dwek, E., \& Woosley, S. E. 1977, ApJ, 214, 300

Clayton, D. D., \& Leising, M. D. 1987, Phys. Rep., 144, 1

Clegg, R., Lambert, D. L., \& Bell, R. A. 1979, ApJ, 234, 188

Cosner, K. R., \& Truran, J. W. 1981, Ap\&SS, 78, 85

Diehl, R., et al. 1993, in AIP Conf. Proc. 280, Compton Gamma-Ray Observatory, ed. M. Friedlander, N. Gehrels, \& D. J. Macomb (New York: AIP), 60

Dominy, J. F., \& Wallerstein, G. 1986, ApJ, 310, 371

Forestini, M., Goriely, S., Jorissen, A., \& Arnould, M. 1992, A\&A, 252, 597

Forestini, M., Paulus, G., \& Arnould, M. 1991, A\&A, 252, 597

Fuller, G. M., Fowler, W. A., \& Newman, M. J. 1982, ApJS, 48, 279

Gallino, R., Busso, M., Picchio, G., Raiteri, C. M., \& Renzini, A. 1988, ApJ, 334, L45

Gallino, R., Raiteri, C. M., \& Busso, M. 1993, ApJ, 410, 400

Gallino, R., Raiteri, C. M. Busso, M., \& Matteucci, F. 1994, ApJ, in press

Hollowell, D. E., \& Iben, I., Jr. 1988, ApJ, 333, L25

Hollowell, D. E., \& Iben, I.,

Hoppe, P., Amari, S., Zinner, E., Ireland, T. R., \& Lewis, R. S. 1994, ApJ, in press

Huey, J. M., \& Kohman, T. P. 1972, Earth Planet. Sci. Lett., 16, 401

Huss, G. R., Hutcheon, I. D., \& Wasserburg, G. J. 1993, Lunar Planet. Sci.

Conf. XXIV (Houston: Lunar and Planetary Inst., USRA), 687

Iben, I., Jr. 1983, ApJ, 275, L65

Iben, I. Jr., \& Renzini, A. 1982, ApJ, 263, L23

Iliadis, C., Schange, T., Rolfs, C., Schröder, V., Somorjal, E., Trautvetter, H. P. \& Wolfe, K. 1990, Nucl. Phys., A512, 509

Jeffrey, P. M., \& Reynolds, J. H. 1961, J. Geophys. Res., 66, 3582

Kaiser, T., \& Wasserburg, G. J. 1983, Geochim, Cosmochim. Acta, 47, 43

Käppeler, F., Beer, H., \& Wisshak, K. 1989, Rep. Progr. Phys., 52, 945

Käppeler, F., Gallino, R., Busso, M., Picchio, G., \& Raiteri, C. M. 1990, ApJ, 354,630

Kelly, W. R., \& Wasserburg, G. J. 1978, Geophys. Res. Lett., 5, 1079

Kipper, T. 1991, in Evolution of Stars: the Photospheric Abundance Connection, ed. G. Michaud \& A. Tutukov (Dordrecht: Kluwer), 317

\section{REFERENCES}

Lambert, D. L. 1991, in Evolution of Stars: the Photospheric Abundance Connection, ed. G. Michaud \& A. Tutukov (Dordrecht: Kluwer), 299

Lattanzio, J. C. 1986, ApJ, 311, 708

. 1993, private communication

Lederer, C. M., \& Shirley, V. S. 1978, Tables of Isotopes (New York: Wiley)

Lee, T., Papanastassiou, D. A., \& Wasserburg, G. J. 1977, ApJ, 211, L107

Mahoney, W. A., Ling, J. C., Wheaton, W. A., \& Jacobsen, A. S. 1984, ApJ, 286, 578

McCulloch, M. T., \& Wasserburg, G. J. 1978, ApJ, 220, L15

Merrill, P. W. 1952, Science, 115, 484

Ostic, R. G., El-Badry, H. M., \& Kohman, T. P. 1969, Earth Planet. Sci. Lett., 7,72

Podosek, F. A., \& Swindle, T. D. 1988, in Meteorites and the Early Solar System, ed. J. F. Kerridge \& M. S. Matthews (Tucson: Univ. Arizona Press), 1093

Prantzos, N. 1991, in Gamma-Ray Line Astrophysics, ed. P. G. Durouchoux \& N. Prantzos (New York: American Institute of Physics), 129

Reimers, D. 1975, in Problems in Stellar Atmospheres and Envelopes, ed. B. Baschek, H. Kegel, \& G. Traving (Berlin: Springer), 229

Renzini, A. 1982, ApJ, 211, L107

Renzini, A., \& Voli, M. 1981, A\&A, 94, 175

Reynolds, J. 1960, Phys. Rev. Lett., 4,8

Safronov, V. S. 1969, Evolution of the Protoplanetary Cloud and the Formation of the Earth and Planets (Moscow: Nauka Press), 206

Schönfelder, V. 1993, in Nuclei in the Cosmos, ed. F. Käppeler \& K. Wisshak (Bristol: Inst. Physics Publ.), 97

Schramm, D. N., \& Wasserburg, G. J. 1970, ApJ, 162, 57

Share, G. H., Kinzer, R. L., Kurfess, J. D., Forrest, D. J., Chupp, E. L., \& Rieger, E. 1985, ApJ, 292, L61

Shukolyukov, A., \& Lugmair, G. W. 1993, Science, 259, 1138

Smith, V. V., \& Lambert, D. L. 1985, ApJ, 294, 326 1986, ApJ, 311, 843 1988, ApJ, 333, 219 1990, ApJS, 72, 387

Swindle, T. D., \& Podosek, F. A. 1988, in Meteorites and the Early Solar System, ed. J. F. Kerridge \& M. S. Matthews (Tucson: Univ. Arizona Press), 1127

Takahashi, K., \& Yokoi, K. 1987, Atomic Data Nucl. Data, 36, 375

Tomkin, J., \& Lambert, D. L. 1979, ApJ, 227, 209

Trautvetter, H. P., et al. 1989, Z. Phys. A, 323, 1

Truran, J. W., \& Iben, I., Jr. 1977, ApJ, 216, 797

Truran, J. W., \& Cameron, A. G. W. 1978, ApJ, 219, 226

Ulrich, R. K. 1973, in Explosive Nucleosynthesis, ed. D. N. Schramm \& W. D. Arnett (Austin: Univ. Texas Press), 139

Utsumi, K. 1985, in Cool Stars with Excesses of Heavy Elements, ed. M. Jashek \& P. C. Keenan (Dordrecht : Reidel), 243

Vogelaar, B. 1989, Ph.D. thesis, Caltech

Ward, R. A., \& Fowler, W. A. 1980, ApJ, 238, 266

Wasserburg, G. J. 1985, in Protostars and Planets II, ed. D. C. Black \& M. S Matthews (Tucson: Univ. Arizona Press), 703

Wasserburg, G. J., \& Arnould, M. 1987, in Lecture Notes in Physics 287, 4th Workshop on Nuclear Astrophysics, ed. W. Hillebrant et al. (Berlin: Springer), 262

Wasserburg, G. J., Gallino, R., Busso, M., \& Raiteri, C. M. 1993, Lunar Plant. Sci. Conf. XXIV (Houston: Lunar and Planetary Inst., USRA), 1487

Wasserburg, G. J., Papanastassiou, D. A., \& Lee, T. 1979, in Proc. 22nd Internat. Astrophys. Symp. (Liège: Univ. Liège), 203

Woosley, S. E., Fowler, W. A., Holmes, J. A., \& Zimmerman, B. A. 1978 Atomic Data Nucl. Data, 22, 371

Yokoi, K., Takahashi, K., \& Arnould, M. 1985, A\&A, 145, 339

Zinner, E., Amari, S., Anders, E., \& Lewis, R. S. 1991, Nature, 349, 51

Zook, A. C. 1985, ApJ, 289, 356 